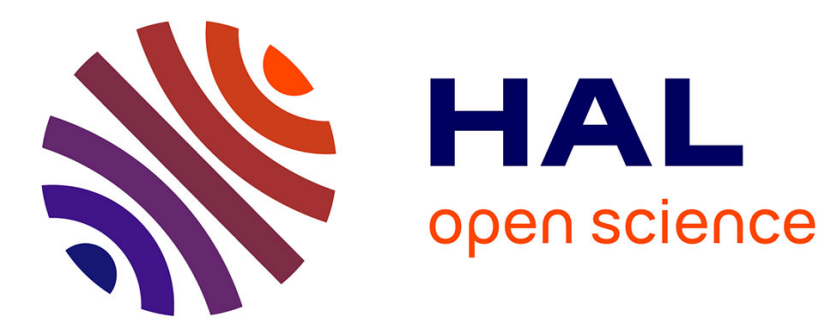

\title{
An optimal IV technique for identifying continuous-time transfer function model of multiple input systems
}

\author{
Hugues Garnier, Marion Gilson, Peter C. Young, Eric Huselstein
}

\section{To cite this version:}

Hugues Garnier, Marion Gilson, Peter C. Young, Eric Huselstein. An optimal IV technique for identifying continuous-time transfer function model of multiple input systems. Control Engineering Practice, 2007, 15 (4), pp.471-486. 10.1016/j.conengprac.2006.09.004 . hal-00151323

\section{HAL Id: hal-00151323 \\ https://hal.science/hal-00151323}

Submitted on 4 Jun 2007

HAL is a multi-disciplinary open access archive for the deposit and dissemination of scientific research documents, whether they are published or not. The documents may come from teaching and research institutions in France or abroad, or from public or private research centers.
L'archive ouverte pluridisciplinaire HAL, est destinée au dépôt et à la diffusion de documents scientifiques de niveau recherche, publiés ou non, émanant des établissements d'enseignement et de recherche français ou étrangers, des laboratoires publics ou privés. 
Final version accepted to

Control Engineering Practice

Vol. 15 (4): 471-486, 2007.

DOI link: http://dx.doi.org/10.1016/j.conengprac.2006.09.004

\title{
An optimal IV technique for identifying continuous-time transfer function model of multiple input systems
}

\author{
H. Garnier ${ }^{\ddagger 1}$, M. Gilson ${ }^{\ddagger}$, P.C. YounG ${ }^{\dagger *}$, E. Huselstein ${ }^{\ddagger}$
}

\begin{abstract}
An instrumental variable method for continuous-time model identification is proposed for multiple input single output systems where the characteristic polynomials of the transfer functions associated with each input are not constrained to be identical. An associated model order determination procedure is shown to be reasonably successful. Monte Carlo simulation analysis are used to demonstrate the properties and general robustness of the model order selection and parameter estimation schemes. The results obtained to model a winding process and an industrial binary distillation column illustrate the practical applicability of the proposed identification scheme.
\end{abstract}

Keywords: distillation column; continuous-time systems; identification; instrumental variable; linear differential equation; multiple input systems; parameter estimation; sampled data; winding process.

¥ Centre de Recherche en Automatique de Nancy (CRAN UMR 7039), Nancy-Université, CNRS, BP 239, F-54506 Vandœuvre-lès-Nancy Cedex, France, \{hugues.garnier, marion.gilson\}@cran. uhp-nancy.fr

$\dagger \quad$ Centre for Research on Environmental Systems and Statistics, Lancaster University, Lancaster LA1 4YQ, U.K., p.young@lancaster.ac.uk

* Centre for Resource and Environmental Studies, Australian National University, Canberra, Australia.

\footnotetext{
${ }^{1}$ Corresponding author
} 


\section{Introduction}

System identification is an established field in the area of systems analysis and control. It aims at determining mathematical models for dynamical systems based on observed inputs and outputs. Although dynamical systems in the physical world are normally formulated in the continuous-time (CT) domain, as differential equations, most system identification schemes have been based in the past on discrete-time (DT) models without concern for the merits of the more natural continuoustime models. The development of CT model identification techniques originated in the last century (see e.g. (Young 1970) which adumbrates the methodology described in the present paper) but was overshadowed by the overwhelming developments of DT model identification methods. This was mainly due to the 'go completely digital' trend that was spurred by parallel developments in digital computers. Interest in CT approaches to system identification has however been growing in the recent years (Garnier et al. 2003), (Li et al. 2003), (Wang et al. 2004), (Garnier and Young 2004), (Moussaoui et al. 2005), (Mensler et al. 2006), (Young and Garnier 2006), (Mahata and Garnier 2006), (Rao and Unbehauen 2006), (Garnier and Wang 2007).

In this paper, a new identification method is developed for multiple input single output (MISO) continuous-time linear systems. In DT model identification, the approaches dedicated to multiple transfer function model identification combine either extensions of linear regression techniques like pseudo-linear, multi-linear regression, filtering, instrumental variable, or non linear optimization techniques (Ljung 1999). For the CT case, as far as the authors are aware, the only procedure developed to handle the MISO identification problem is based on non linear optimization techniques which minimize the output error. However, the technique may critically rely on a good initial parameter set to converge to the global minimum of the cost function. The linear regression-based algorithms should offer an interesting solution to overcome this drawback. However, the parameter estimation procedures for MISO systems have usually been developed by a straightforward extension of procedures devoted to SISO systems, which only allows transfer function estimation with a common denominator. Since this case is not very realistic in many practical applications, this paper presents a new method to estimate MISO systems described by multiple CT transfer functions with different denominators.

When looking at methods that can consistently identify systems while relying on simple linear regression algorithms, instrumental variable (IV) techniques seem to be rather attractive ((Söderström and Stoica 1983), (Young 1984) or (Gilson and Van den Hof 2005) for a recent reference). Moreover, when dealing with highly complex processes that are high dimensional in terms of inputs and outputs, it can be rather attractive to rely on methods that do not require non-convex optimization.

Several IV estimators have been developed for CT SISO system identification (Garnier et al. 2003). Amongst these, the Simplified Refined Instrumental Variable for Continuous-time systems (Young and Jakeman 1980), denoted by SRIVC from hereon, presents the advantage of yielding asymptotically efficient estimates in the presence of white measurement noise. Therefore, the main objective in this paper is to develop a SRIVC version dedicated to CT multiple transfer function model identification, which is a CT version of a similar SRIV algorithm for DT systems (Young and Jakeman 1979), (Jakeman et al. 1980). Another interesting advantage of using this refined IV method is that a procedure based on the properties of the instrumental product matrix (Young et al. 1980) can be used for identifying the model structure prior to parameter estimation.

The paper is organized in the following way. Section 2 states the problem. The proposed method is described in Section 3. The properties of the proposed algorithm are illustrated through Monte Carlo simulation in Section 4. The robustness of the proposed estimation scheme against the initial parameter set is also illustrated and compared with a traditional output error technique. Sections 5 and 6 present the results of the identification of a winding process and an industrial 
binary distillation column respectively. Finally, Section 7 gives some concluding remarks.

\section{Problem statement}

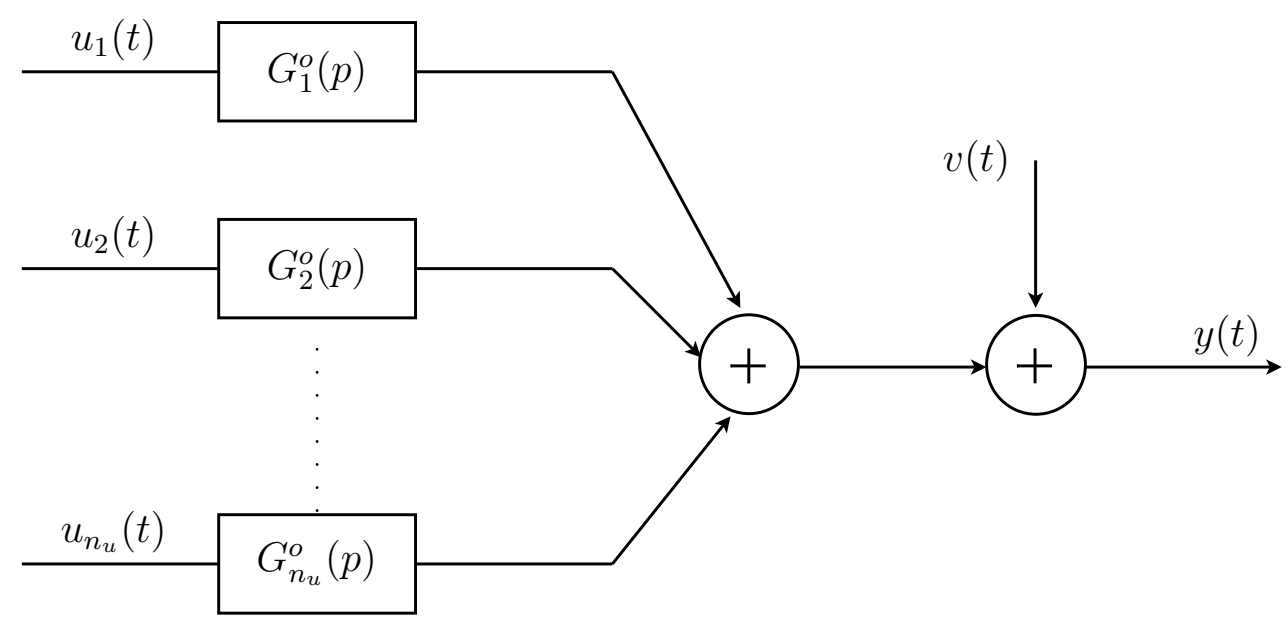

Figure 1: Multiple input system to be identified

Consider a MISO CT linear time-invariant causal system that can be described by (see Figure 1)

$$
\mathcal{S}:\left\{\begin{array}{l}
y_{u_{i}}(t)=G_{i}^{o}(p) u_{i}\left(t-\tau_{i}^{o}\right), \\
y_{u}(t)=\sum_{i=1}^{n_{u}} y_{u_{i}}(t), \\
y(t)=y_{u}(t)+v(t),
\end{array}\right.
$$

with

$$
\begin{aligned}
G_{i}^{o}(p) & =\frac{B_{i}^{o}(p)}{F_{i}^{o}(p)} \\
B_{i}^{o}(p) & =b_{i, 0}^{o}+b_{i, 1}^{o} p+\ldots+b_{i, m_{i}}^{o} p^{m_{i}}, \\
F_{i}^{o}(p) & =f_{i, 0}^{o}+f_{i, 1}^{o} p+\ldots+f_{i, n_{i}}^{o} p^{n_{i}}, \\
f_{i, n_{i}}^{o} & =1, \quad n_{i} \geq m_{i}, \quad i=1, \ldots, n_{u},
\end{aligned}
$$

where $u(t)=\left[u_{1}(t) \ldots u_{n_{u}}(t)\right]$ is the vector of uncorrelated input signals, $y_{u}(t)$ the system response to $u(t)$ and $v(t)$ is the disturbance signal ; $p$ is the differential operator, i.e. $p x(t):=\frac{d x(t)}{d t} ; \tau_{i}^{o}$ denotes the time-delay between the output and the $i$ th corresponding input. The polynomials $F_{i}^{o}(p)$ and $B_{i}^{o}(p)$ are assumed to be relatively prime and the roots of the polynomials $F_{i}^{o}(p)$ are assumed to have negative real parts; the system under study is therefore assumed to be asymptotically stable.

The first equation in (1) describes the $i$ th output at all values of the continuous-time variable $t$ and can also be written as

$$
f_{i, 0}^{o} y_{u_{i}}(t)+f_{i, 1}^{o} y_{u_{i}}^{(1)}(t)+\cdots+y_{u_{i}}^{\left(n_{i}\right)}(t)=b_{i, 0}^{o} u_{i}\left(t-\tau_{i}^{o}\right)+\cdots+b_{i, m_{i}}^{o} u_{i}^{\left(m_{i}\right)}\left(t-\tau_{i}^{o}\right)
$$

where $x^{(l)}(t)$ denotes the $l$ th time-derivative of the continuous-time signal $x(t)$. The system is subject to an arbitrary set of initial conditions

$$
\begin{aligned}
u^{0} & =\left[\begin{array}{lll}
u_{1}^{0} & \cdots & u_{n_{u}}^{0}
\end{array}\right], y_{u}^{0}=\left[\begin{array}{lll}
y_{u_{1}}^{0} & \cdots & y_{u_{n_{u}}}^{0}
\end{array}\right], \\
u_{i}^{0} & =\left[\begin{array}{llll}
u_{i}(0) & u_{i}^{(1)}(0) & \cdots & u_{i}^{\left(m_{i}-1\right)}(0)
\end{array}\right]^{T} \in \mathbb{R}^{m_{i}}, \\
y_{u_{i}}^{0} & =\left[\begin{array}{llll}
y_{u_{i}}(0) & y_{u_{i}}^{(1)}(0) & \cdots & y_{u_{i}}^{\left(n_{i}-1\right)}(0)
\end{array}\right]^{T} \in \mathbb{R}^{n_{i}} .
\end{aligned}
$$


It is furthermore assumed that the disturbances that cannot be explained from the input signal can be lumped into the additive term $v(t)(1)$. The disturbance term $v(t)$ is assumed to be independent of the inputs $u_{i}(t)$, i.e. the case of the open-loop operation of the system is considered. For the identification problem, it is also assumed that the continuous-time signals $u_{i}(t)$ and $y(t)$ are sampled at regular time-interval $T_{s}$.

The goal is then to build a model of equation (1) based on sampled input and output data. Models of the following form are considered

$$
\mathcal{G}:\left\{\begin{array}{l}
y_{u_{i}}\left(t_{k}, \theta_{i}\right)=G_{i}\left(p, \theta_{i}\right) u_{i}\left(t_{k}-\tau_{i}\right) \\
y_{u}\left(t_{k}, \theta_{i}\right)=\sum_{i=1}^{n_{u}} y_{u_{i}}\left(t_{k}, \theta_{i}\right), \\
y\left(t_{k}\right)=y_{u}\left(t_{k}, \theta_{i}\right)+v\left(t_{k}\right)
\end{array}\right.
$$

where $x\left(t_{k}\right)$ denotes the sample of the continuous-time signal $x(t)$ at time-instant $t=k T_{s}$ and $G_{i}\left(p, \theta_{i}\right)$ is the $i$ th transfer function given by

$$
\begin{aligned}
G_{i}\left(p, \theta_{i}\right) & =\frac{B_{i}(p)}{F_{i}(p)}=\frac{b_{i, 0}+b_{i, 1} p+\cdots+b_{i, m_{i}} p^{m_{i}}}{f_{i, 0}+f_{i, 1} p+\cdots+f_{i, n_{i}} p^{n_{i}}}, \\
f_{i, n_{i}} & =1, \quad n_{i} \geq m_{i}, \quad i=1, \ldots, n_{u},
\end{aligned}
$$

and $\theta_{i}=\left[\begin{array}{llllll}b_{i, m_{i}} & \ldots & b_{i, 0} & f_{i, n_{i}-1} & \ldots & f_{i, 0}\end{array}\right]^{T} \in \mathbb{R}^{n_{p_{i}}}$, with $n_{p_{i}}=n_{i}+m_{i}+1$, where $n_{i}$ and $m_{i}$ denote the denominator and numerator orders of $G_{i}\left(p, \theta_{i}\right)$ respectively. Therefore, the sought parameter vector is

$$
\theta=\left[\begin{array}{lll}
\theta_{1}^{T} & \ldots & \theta_{n_{u}}^{T}
\end{array}\right]^{T} \in \mathbb{R}^{n_{p} \times 1}
$$

with $n_{p}=\sum_{i=1}^{n_{u}} n_{p_{i}}$.

Note that estimation methods presented in this paper focus on identifying the parameters of each plant transfer function $G_{i}\left(p, \theta_{i}\right)$ rather than the additive noise appearing in (1). The disturbance term is assumed here to be a zero-mean discrete-time noise sequence denoted as $v\left(t_{k}\right)$. Moreover, the pure time-delays are supposed to be known and multiple integers of the sampling period $\tau_{i}=n_{k_{i}} T_{s}$.

The identification problem can now be stated as follows: determine the orders $\left(n_{i}\right.$ and $\left.m_{i}\right)$ and the parameter vector $\theta=\left[\theta_{1}^{T} \cdots \theta_{n_{u}}^{T}\right]^{T}$ of the continuous-time plant model from $N$ sampled measurements of the input and the output $Z^{N}=\left\{y\left(t_{k}\right) u_{1}\left(t_{k}\right) \ldots u_{n_{u}}\left(t_{k}\right)\right\}_{k=1}^{N}$.

\section{Refined IV methods for continuous-time transfer func- tion model}

\subsection{Continuous-time transfer function model identification}

There are mainly two time-domain approaches to determine a CT model from sampled data. The first is to estimate a DT model which is then converted into a CT model. The second approach consists in identifying directly a CT model from the DT data. In comparison with the DT counterpart, CT model identification raises several technical issues. The first is related to the fact that unlike the difference equation model, the differential equation model is not a linear combination of samples of only the measurable process input and output signals. It also contains input and output time-derivatives which are not available as measurement data in most practical cases. Various types of continuous-time filters, such as the traditional State-Variable Filter (SVF) method, have been devised to circumvent the need to reconstruct these time-derivatives (Garnier et al. 2003). The CONtinuous-Time System IDentification (CONTSID) toolbox has been developed on the basis of these methods (Garnier et al. 2006). 
Most of these CT model identification methods present the following drawbacks. First, they can handle the case of MISO common denominator (CD) transfer function models only. Secondly, these approaches require the a priori choice of a design parameter which can be difficult from a practical point of view. Thirdly they disregard the properties of the additive noise and therefore represent a sub-optimal solution to the estimation problem. One particularly successful stochastic identification method is the iterative SRIVC method (see (Young and Jakeman 1980), (Young 2002)), where it is referred to as RIVC). This approach involves a method of adaptive prefiltering based on an optimal ${ }^{2}$ statistical solution to the problem when the additive noise $v\left(t_{k}\right)$ is white, but which also yields consistent and relatively low variance parameter estimates in the case of coloured noise. This estimation technique was first proposed for DT model identification in the form of the Refined Instrumental Variable (RIV) algorithm ${ }^{3}$ (Young 1976), (Young 1984) and then extended for DT MISO systems with different denominators (Jakeman et al. 1980). The CT MISO algorithm described in the present paper uses the same type of iterative, relaxation algorithm as that used in this DT MISO algorithm. The RIV approach was extended for SISO CT model identification at the time of its original development (Young and Jakeman 1980). It has recently been revisited (Young 2002) and adapted to handle the case of irregularly sampled data (Huselstein and Garnier 2002) (see also (Raghavan et al. 2006) for a recent review of identification approaches for handling irregularly sampled data). This IV-type of method has often proved to be particularly useful in practical applications (see e.g. (Young 1998)). This method not only ensures that the estimate converges to statistically optimum values in the case of additive white noise, it also generates information on the parametric error covariance matrix which can be used in an associated procedure to identify the orders of the component transfer function models. SRIVC is also a logical extension of the traditional and more heuristically defined SVF approach but presents the advantage of not requiring manual specification of prefilter parameters.

In the following section, the SRIVC version for SISO transfer function model is first briefly recalled and then extended to handle the case of multiple transfer function models.

\subsection{SRIVC for SISO transfer function models}

Consider a SISO system with a white measurement noise on the output. The SRIVC method is based on the Maximum Likelihood (ML) approach where the error function is given by the output error

$$
v\left(t_{k}, \theta\right)=y\left(t_{k}\right)-\frac{B(p, \theta)}{F(p, \theta)} u\left(t_{k}-\tau\right)
$$

with

$$
F(p, \theta)=\sum_{l=0}^{n-1} f_{l} p^{l}+p^{n} \quad \text { and } B(p, \theta)=\sum_{l=0}^{m} b_{l} p^{l} .
$$

Minimisation of a least squares criterion function in $v\left(t_{k}, \theta\right)$ provides the basis for the output error estimation methods. However, $v\left(t_{k}, \theta\right)$ can also be rewritten as

$$
v\left(t_{k}, \theta\right)=\frac{1}{F(p, \theta)}\left[F(p, \theta) y\left(t_{k}\right)-B(p, \theta) u\left(t_{k}-\tau\right)\right]
$$

\footnotetext{
${ }^{2}$ Strictly, the method is quasi-optimal because true optimality would require optimal interpolation of the input signal $u(t)$ over the sampling interval, whereas only simple interpolation is used in the SRIVC implementation. However, this normally produces very good, near optimal estimation results.

${ }^{3}$ The RIV algorithm for DT model identification is available in the CAPTAIN toolbox (see http://www.es.lancs.ac.uk/cres/captain/).
} 
Therefore, the output error given in (12) can be transformed in this manner to yield an equation error expression of the form,

$$
v\left(t_{k}, \theta\right)=F(p, \theta) \tilde{y}\left(t_{k}\right)-B(p, \theta) \tilde{u}\left(t_{k}-\tau\right)
$$

where $\tilde{y}\left(t_{k}\right)$ and $\tilde{u}\left(t_{k}\right)$ are the variables pre-filtered by $L(p, \theta)=\frac{1}{F(p, \theta)}$. The problem with this formulation is that $\theta$ and therefore $F(p, \theta)$ are unknown a priori. This problem can be conveniently solved by employing an iterative optimization procedure which aims at adjusting an initial estimate $\theta^{0}$ of $\theta$ adaptively until it converges on an optimal estimate. Therefore, at each step, a linear in the unknown parameter vector $\theta$ equation has to be solved

$$
\tilde{y}^{(n)}\left(t_{k}, \hat{\theta}^{j}\right)=\tilde{\phi}^{T}\left(t_{k}, \hat{\theta}^{j}\right) \theta^{j+1}+\varepsilon\left(t_{k}, \hat{\theta}^{j}\right)
$$

where $\hat{\theta}^{j}$ is the parameter vector estimated at the $j$ th step of the algorithm, $\theta^{j+1}$ is the parameter vector to be estimated and

$$
\tilde{\phi}^{T}\left(t_{k}, \hat{\theta}^{j}\right)=\left[\tilde{u}^{(m)}\left(t_{k}-\tau, \hat{\theta}^{j}\right) \ldots \tilde{u}\left(t_{k}-\tau, \hat{\theta}^{j}\right)-\tilde{y}^{(n-1)}\left(t_{k}, \hat{\theta}^{j}\right) \ldots-\tilde{y}\left(t_{k}, \hat{\theta}^{j}\right)\right],
$$

where

$$
\tilde{x}^{(i)}\left(t_{k}, \hat{\theta}^{j}\right)=\frac{p^{i}}{F\left(p, \hat{\theta}^{j}\right)} \tilde{x}\left(t_{k}, \hat{\theta}^{j}\right)
$$

The use of the conventional least squares method to solve (16) will give biased results when the output measurement is corrupted by noise. A solution is to use an IV-type of method to overcome the bias problem. However, the choice of the instruments, denoted by $\tilde{Z}^{T}\left(t_{k}\right)$ here, was shown to have considerable effect on the parametric covariance matrix $P_{i}$. The lower bound of $P_{i}$ for any unbiased identification method is given by the Cramer-Rao bound, which is specified (see e.g. (Ljung 1999) and (Söderström and Stoica 1983))

$$
P_{i} \geq P_{i}^{o p t}
$$

with $^{4}$

$$
P_{i}^{o p t}=\sigma_{\varepsilon}^{2}\left[\overline{\mathbb{E}} \overline{\tilde{\phi}}\left(t_{k}\right) \overline{\tilde{\phi}}^{T}\left(t_{k}\right)\right]^{-1}
$$

where $\overline{\tilde{\phi}}\left(t_{k}\right)=L(p) \bar{\phi}\left(t_{k}\right)$ and $\bar{\phi}\left(t_{k}\right)$ is the noise-free part of $\phi\left(t_{k}\right)$. The minimum variance can then be achieved by the following choice of design variables (see (Young and Jakeman 1980)) where these filters are defined by a special maximum likelihood solution to the problem):

$$
\left\{\begin{array}{l}
L(p)=\frac{1}{F^{o}(p)} \\
\tilde{Z}^{T}\left(t_{k}\right)=\frac{1}{F^{o}(p)} \bar{\phi}\left(t_{k}\right)
\end{array}\right.
$$

Since the exact model $F^{o}(p)$ is not known, it has to be replaced by its estimate obtained at the previous iteration: i.e.,

$$
\left\{\begin{array}{l}
L\left(p, \hat{\theta}^{j}\right)=\frac{1}{F\left(p, \hat{\theta}^{j}\right)} \\
\tilde{Z}^{T}\left(t_{k}, \hat{\theta}^{j}\right)=\left[\tilde{u}^{(m)}\left(t_{k}-\tau, \hat{\theta}^{j}\right) \ldots \tilde{u}\left(t_{k}-\tau, \hat{\theta}^{j}\right)-\tilde{y}_{u}^{(n-1)}\left(t_{k}, \hat{\theta}^{j}\right) \ldots-\tilde{y}_{u}\left(t_{k}, \hat{\theta}^{j}\right)\right]
\end{array}\right.
$$

\footnotetext{
${ }^{4}$ The notation $\overline{\mathbb{E}}[]=.\lim _{N \rightarrow \infty} \frac{1}{N} \sum_{k=0}^{N-1} \mathbb{E}[$.$] is adopted from the prediction error framework of (Ljung 1999).$
} 
where the filtered auxiliary model output is obtained from

$$
\tilde{y}_{u}\left(t_{k}, \hat{\theta}^{j}\right)=\frac{B\left(p, \hat{\theta}^{j}\right)}{F\left(p, \hat{\theta}^{j}\right)} \tilde{u}\left(t_{k}-\tau\right)
$$

$\tilde{Z}^{T}\left(t_{k}, \hat{\theta}^{j}\right)$ is thus an estimation of the filtered noise-free part of the regressor $\phi\left(t_{k}\right)$. The optimal IV-based parameter estimates are then given by

$$
\hat{\theta}^{j+1}=\left[\sum_{k=1}^{N} \tilde{Z}\left(t_{k}, \hat{\theta}^{j}\right) \tilde{\phi}^{T}\left(t_{k}, \hat{\theta}^{j}\right)\right]^{-1} \sum_{k=1}^{N} \tilde{Z}^{T}\left(t_{k}, \hat{\theta}^{j}\right) \tilde{y}^{(n)}\left(t_{k}, \hat{\theta}^{j}\right),
$$

The main steps of the SRIVC algorithm dedicated to $\mathrm{SISO}^{5}$ transfer function model are presented in (Young and Jakeman 1980) and (Young 2002). It may be noted that since the instruments are correlated with the input/output data but uncorrelated with the noise, the proposed IV algorithm delivers consistent parameters even if the additive noise is a colored noise process. However, it only gives asymptotically efficient estimates in the case of a white noise. In practical situations, the additive noise will not have the nice white noise properties assumed above: it is likely that the noise will be a colored noise process $v(t)=H^{o}(p) e(t)$. In such a case, (21) becomes

$$
\left\{\begin{array}{l}
L(p)=\frac{1}{H^{o}(p) F^{o}(p)} \\
\tilde{Z}^{T}\left(t_{k}\right)=\frac{1}{H^{o}(p) F^{o}(p)} \bar{\phi}\left(t_{k}\right)
\end{array}\right.
$$

The exact noise model is unknown in practice but could be estimated by extending to the continuous-time case the procedure used in the full discrete-time RIV version (see (Young 1984) or (Jakeman et al. 1980)) where an AR or ARMA model for the noise part is estimated and used in the prefiltering operation. This would lead to the identification of a hybrid model where the plant model would be in continuous-time while the noise part would be in discrete-time, as suggested recently (Young et al. 2006).

\subsection{SRIVC for multiple transfer function models}

The proposed method derives from the equivalent iterative, relaxation algorithm for DT models in (Young and Jakeman 1980) and (Jakeman et al. 1980). It aims at identifying MISO model with different denominators (DD) for each input (9), which is more realistic than assuming an identical denominator for all transfer function. However, the model is no longer linear in the parameters and the proposed MISO version of SRIVC lies, therefore, in the domain of multi-linear regression. The MISO model (9) can be converted into $n_{u}$ SISO models as follows

$$
\begin{aligned}
& v_{i}\left(t_{k}, \theta\right)=\tilde{\xi}_{i}\left(t_{k}, \theta\right)-\tilde{y}_{u_{i}}\left(t_{k}, \theta_{i}\right) \quad \text { for } i=1 \ldots n_{u} \\
& \tilde{\xi}_{i}\left(t_{k}, \theta\right)=\tilde{y}\left(t_{k}\right)-\sum_{j=1, j \neq i}^{n_{u}} \tilde{y}_{u_{j}}\left(t_{k}, \theta_{j}\right)
\end{aligned}
$$

The parameter vector $\theta$ is partitioned ${ }^{6}$ into classes $\theta_{1}, \ldots, \theta_{n_{u}}$ such that the error is affine with respect to the parameters of any of these classes when the parameters of all others are fixed (Walter

\footnotetext{
${ }^{5}$ The case of multiple transfer function models with common denominators can be handled in a straightforward manner.

${ }^{6}$ The partition of $\theta$ into sub-vectors $\theta_{i}$ is related to the model order problem discussed further in Section 3.4 .
} 
and Pronzato 1997). It is then possible to search for $\hat{\theta}$ by applying successively the SISO version of the SRIVC algorithm to estimate the parameters of each class in turn, with a cyclic exploration of all classes. This is achieved by following the same type of 'relaxation' procedure described in Section 3.2

$$
\begin{aligned}
& \tilde{\xi}_{i}^{\left(n_{i}\right)}\left(t_{k}, \hat{\theta}^{j}\right)=\tilde{\phi}_{i}^{T}\left(t_{k}, \hat{\theta}^{j}\right) \theta_{i}^{j+1}+\tilde{\varepsilon}_{i}\left(t_{k}, \hat{\theta}^{j}\right)
\end{aligned}
$$

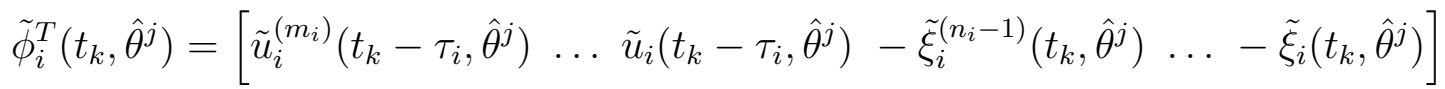

where the filter is $L_{i}\left(p, \hat{\theta}_{i}^{j}\right)=\frac{1}{F_{i}\left(p, \hat{\theta}_{i}^{j}\right)}$. This equation is then solved by using the IV estimator described in the previous section.

The main steps of the proposed iterative SRIVC method can be summarized by the following algorithm ${ }^{7}$.

1. Estimate the initial parameter vectors

$$
\hat{\theta}_{i}^{0}=\left[\begin{array}{llllll}
\hat{b}_{i, m_{i}}^{0} & \ldots & \hat{b}_{i, 0}^{0} & \hat{f}_{i, n_{i}-1}^{0} & \ldots & \hat{f}_{i, 0}^{0}
\end{array}\right]^{T} \text { for } i=1 \ldots n_{u}
$$

between the output $y\left(t_{k}\right)$ and each input $u_{i}\left(t_{k}\right)$.

Calculate the auxiliary model outputs

$$
y_{u_{i}}\left(t_{k}, \hat{\theta}_{i}^{0}\right)=\frac{B_{i}\left(p, \hat{\theta}_{i}^{0}\right)}{F_{i}\left(p, \hat{\theta}_{i}^{0}\right)} u_{i}\left(t_{k}-\tau_{i}\right)
$$

2. $j=0 \ldots N_{\text {iter }}-1, i=1 \ldots n_{u}$

(a) Generate an estimate $\xi_{u_{i}}\left(t_{k}, \hat{\theta}_{i}^{j}\right)$ of the noisy response to $u_{i}$,

$$
\xi_{u_{i}}\left(t_{k}, \hat{\theta}_{i}^{j}\right)=y\left(t_{k}\right)-\sum_{l=1, l \neq i}^{n_{u}} y_{u_{l}}\left(t_{k}, \hat{\theta}_{l}^{j}\right) .
$$

Filter the latter variable, the input signal and the auxiliary model output

$$
\begin{gathered}
\tilde{\xi}_{u_{i}}\left(t_{k}, \hat{\theta}_{i}^{j}\right)=\frac{1}{F_{i}\left(p, \hat{\theta}_{i}^{j}\right)} \xi_{u_{i}}\left(t_{k}, \hat{\theta}_{i}^{j}\right), \\
\tilde{u}_{i}\left(t_{k}, \hat{\theta}_{i}^{j}\right)=\frac{1}{F_{i}\left(p, \hat{\theta}_{i}^{j}\right)} u_{i}\left(t_{k}\right), \\
\tilde{y}_{u_{i}}\left(t_{k}, \hat{\theta}_{i}^{j}\right)=\frac{1}{F_{i}\left(p, \hat{\theta}_{i}^{j}\right)} y_{u_{i}}\left(t_{k}, \hat{\theta}_{i}^{j}\right) .
\end{gathered}
$$

(b) Build up the regressor (29) and the instruments

$$
\tilde{Z}_{i}^{T}\left(t_{k}, \hat{\theta}_{i}^{j}\right)=\left[\tilde{u}_{i}^{\left(m_{i}\right)}\left(t_{k}-\tau_{i}, \hat{\theta}_{i}^{j}\right) \ldots \tilde{u}_{i}\left(t_{k}-\tau_{i}, \hat{\theta}_{i}^{j}\right)-\tilde{\xi}_{u_{i}}^{\left(n_{i}-1\right)}\left(t_{k}, \hat{\theta}_{i}^{j}\right) \ldots-\tilde{\xi}_{u_{i}}\left(t_{k}, \hat{\theta}_{i}^{j}\right)\right] .
$$

Calculate the IV estimate of the parameter vector $\hat{\theta}_{i}^{j+1}$

$$
\hat{\theta}_{i}^{j+1}=\left[\sum_{k=1}^{N} \tilde{Z}_{i}\left(t_{k}, \hat{\theta}_{i}^{j}\right) \tilde{\phi}_{i}^{T}\left(t_{k}, \hat{\theta}_{i}^{j}\right)\right]^{-1} \sum_{k=1}^{N} \tilde{Z}_{i}^{T}\left(t_{k}, \hat{\theta}_{i}^{j}\right) \tilde{\xi}_{i}^{\left(n_{i}\right)}\left(t_{k}, \hat{\theta}_{i}^{j}\right) .
$$

${ }^{7}$ This can also be considered as a 'backfitting' algorithm: see (Young et al. 2001), where a similar device is used to identify nonlinear, state-dependent parameter systems. 
Use $\hat{\theta}_{i}^{j+1}$ to generate the auxiliary model output

$$
y_{u_{i}}\left(t_{k}, \hat{\theta}_{i}^{j+1}\right)=\frac{B_{i}\left(p, \hat{\theta}_{i}^{j+1}\right)}{F_{i}\left(p, \hat{\theta}_{i}^{j+1}\right)} u_{i}\left(t_{k}-\tau_{i}\right) .
$$

Repeat step 2 until the relative error on the parameters is sufficiently small

$$
\sum_{i=1}^{n_{u}} \sum_{l=1}^{n_{p_{i}}}\left|\frac{\hat{\theta}_{i, l}^{j+1}-\hat{\theta}_{i, l}^{j}}{\hat{\theta}_{i, l}^{j}}\right|<\epsilon,
$$

where $\hat{\theta}_{i, l}^{j}$ denotes the $l$ th element of the parameter $\hat{\theta}_{i}^{j}, \epsilon$ is a given tolerance and $N_{i t e r}$ is the final iteration number.

3. For $i=1 \ldots n_{u}, \hat{\theta}_{i}=\hat{\theta}_{i}^{N_{i t e r}}$. Compute an estimate of the parametric covariance matrix $\hat{P}_{i}$ (see (Young 2002) for example)

$$
\hat{P}_{i}=\hat{\sigma}_{\varepsilon}^{2}\left[\sum_{k=1}^{N} \tilde{Z}_{i}\left(t_{k}, \hat{\theta}_{i}\right) \tilde{Z}_{i}^{T}\left(t_{k}, \hat{\theta}_{i}\right)\right]^{-1}
$$

where $\hat{\sigma}_{\varepsilon}^{2}$ denotes the empirical variance of the simulation error

$$
\varepsilon\left(t_{k}, \hat{\theta}\right)=y\left(t_{k}\right)-y_{u}\left(t_{k}, \hat{\theta}\right) .
$$

The parameter vector and parametric covariance matrix estimates are given by

$$
\begin{aligned}
\hat{\theta}= & {\left[\hat{\theta}_{1}^{T} \ldots \hat{\theta}_{n_{u}}^{T}\right]^{T} } \\
\hat{P}= & \left(\begin{array}{cccc}
\hat{P}_{1} & 0 & \cdots & 0 \\
0 & \ddots & \ddots & \vdots \\
\vdots & \ddots & \ddots & 0 \\
0 & \cdots & 0 & \hat{P}_{n_{u}}
\end{array}\right)
\end{aligned}
$$

\section{Remarks}

1. For the initialisation of the algorithm, estimates for a particular transfer function $i$ can be obtained using SISO modeling of the output $y\left(t_{k}\right)$ against each input $u_{i}\left(t_{k}\right)$ in turn. This is not a difficult task and there are several alternatives available to the user in the CONTSID toolbox. An automatic option is to use the DT version (SRIV) of the SRIVC method, which has the advantage of not requiring any design parameters to be specified. The estimated discrete-time model is then first used to generate the auxiliary model outputs $y_{u_{i}}\left(t_{k}, \hat{\theta}_{i}^{0}\right)$ and also converted to continuous-time form to provide the $\hat{F}_{i}\left(p, \hat{\theta}_{i}^{0}\right)$ polynomials. Two alternatives include the user specification of a single cut-off frequency used in either the traditional least squares-based SVF or the basic IV-based GPMF methods (see (Garnier et al. 2003) for example).

2. This method is an IV-type estimation technique. Therefore, upon convergence, it yields consistent estimates, when the model belongs to the system class $\left(G_{o} \in \mathcal{G}^{8}\right)$.

3. The proposed approach can be implemented recursively (Young and Jakeman 1980).

\footnotetext{
${ }^{8}$ This notation is adopted from (Ljung 1999).
} 
4. An indication of the estimated parameter uncertainties is given which makes it possible to assess the model quality. Note, however, that there is an implicit assumption, introduced by the nature of the algorithm, that the parameter estimates of each component $\mathrm{TF}$ are statistically independent (see (36)).

5. The proposed estimation scheme is implemented in both CONTSID $^{9}$ and CAPTAIN $^{10}$ toolboxes for Matlab.

6. If the measurement noise $v\left(t_{k}\right)$ is coloured, then the method is not optimal in statistical terms. However, experience has shown that it is robust and normally yields estimates with reasonable statistical efficiency (i.e. low but not minimum variance). In the colored noise situation, it is possible to use, albeit at the cost of increased complexity, a hybrid approach where the noise modelling, as well as the noise-derived parts of the prefiltering, are carried out in discrete-time terms, as suggested recently (Young et al. 2006).

\subsection{Model order estimation}

A key point to be solved in the identification procedure concerns the model order selection. The method available for SISO systems (see e.g. (Young 1989), (Young 2002)) is extended to the case of MISO systems. While models are estimated from a given data set, two statistical measures are computed and used to choose between a range of model orders. These are $R_{T}^{2}$ and $Y I C$, which are defined as follows,

$$
\begin{aligned}
R_{T}^{2} & =1-\frac{\hat{\sigma}_{\varepsilon}^{2}}{\hat{\sigma}_{y}^{2}}, \\
Y I C & =\log _{e}\left\{\frac{\hat{\sigma}_{\varepsilon}^{2}}{\hat{\sigma}_{y}^{2}}\right\}+\log _{e} \frac{1}{n_{p}} \sum_{i=1}^{n_{u}} \sum_{l=1}^{n_{p_{i}}} \frac{\hat{\sigma}_{\varepsilon}^{2} \hat{\sigma}_{\theta_{i, l}}^{2}}{\hat{\theta}_{i, l}^{2}}
\end{aligned}
$$

where $\hat{\sigma}_{y}^{2}, \hat{\sigma}_{\varepsilon}^{2}$ denote, respectively, the variance of the measured output and the variance of the simulation error; $\hat{\theta}_{i, l}^{2}$ is the squared value of the $l^{\text {th }}$ element of the estimated parameter vector $\hat{\theta}_{i}$; $\hat{\sigma}_{\theta_{i, l}}^{2}$ is the $l$ th diagonal element of the SRIVC estimated parametric covariance matrix $\hat{P}_{i}$; and $n_{p}$ is the total number of parameters. $R_{T}^{2}$ is recognized as the coefficient of determination based on the simulation error. It is a measure of how well the model output explains to the system output and will be close to 1 in low noise situations. However, $R_{T}^{2}$ does not provide a clear indication of the best model order and can suggest over-parameterized models. The Young's Information Criterion $(Y I C)$ is more complex and provides a measure of how well the parameters are defined statistically (see (Young 1996) for example): the more negative the YIC, the better the definition. However it may lead to underestimate the model orders. Both criteria are inspected to find the orders for which $R_{T}^{2}$ is sufficiently high to indicate a good explanation of the data and the YIC is sufficiently negative to indicate well defined parameter estimates.

Note that the proposed procedure, based on the two criteria, is not always completely unambiguous, as all model order procedures and other factors, such as physical considerations and parsimony, need also to be taken into account in the final selection of the model order. However, as illustrated in the next Section, the proposed SRIVC-based model order selection procedure is helpful and is shown to be reasonably successful for the multiple input transfer function model, although the procedure is not as clearly defined as in the equivalent SISO situation.

\footnotetext{
${ }^{9}$ see http://www.cran.uhp-nancy.fr/contsid/

${ }^{10}$ see http://www.es.lancs.ac.uk/cres/captain/
} 


\section{Simulation examples}

Two simulation examples are considered in this section. The system orders are first assumed to be known and Monte Carlo simulations are used to illustrate the relevance of the proposed SRIVC estimation scheme in comparison to the traditional identification method for MISO transfer function model with common denominators. The performance of the proposed approach is also compared with the direct CT model identification method for MISO transfer finction model with different denominators minimizing the Output Error $(\mathrm{COE})^{11}$. The model order selection procedure described in Section 3.4 is then evaluated. The first system has two transfer functions with approximately the same bandwidth; while the second example has two transfer functions with clearly distinguished bandwidths.

\subsection{Simulation example 1}

The first system considered (S1) is a two input, one output system, with second-order nonminimum phase transfer functions

$$
\mathrm{S} 1:\left\{\begin{aligned}
y_{u}(t) & =\frac{-0.5 p+1}{p^{2}+0.6 p+1} u_{1}(t)+\frac{-3 p+2}{p^{2}+4 p+3} u_{2}(t) \\
y\left(t_{k}\right) & =y_{u}\left(t_{k}\right)+v\left(t_{k}\right)
\end{aligned}\right.
$$

The first transfer function presents a resonant mode, with a damping coefficient of 0.3 and a natural frequency of $1 \mathrm{rad} / \mathrm{s}$, while the second has two time constants equal to 1 and $3 \mathrm{~s}$. The dynamic characteristics between the two inputs and the output are quite similar, as can be observed from the step response of both transfer functions displayed in Figure 2(a).

The measured output $y\left(t_{k}\right)$ consists of the noise-free output $y_{u}(t)$ sampled at time $t_{k}$, to which is added a zero-mean independent identically distributed (i.i.d.) Gaussian sequence $v\left(t_{k}\right)$. Note that $\mathrm{S} 1$ is formulated with the model structure assumed in both SRIVC and COE methods. The sampling period is equal to $T_{s}=50 \mathrm{~ms}$. The system is excited by two uncorrelated PRBS of maximum length. The characteristics of the PRBS signals, whose amplitude switches between -1 and +1 , are the following: the number of stages of the shift register is set to $n_{s 1}=6$, the clock period is set to $n_{p 1}=40$ for the first input, while $n_{s 2}=7$ and $n_{p 2}=20$ for the second input. The first input is duplicated and then truncated in order to have the same number of points $N=2540$ for both inputs. Note that the noise-free system response to the PRBS has been calculated exactly at the sampling instances by discretizing the continuous-time transfer function model, assuming a zero-order hold on the inputs.

The variance of the additive noise $v\left(t_{k}\right)$ on the measured output is adjusted in order to obtain a signal-to-noise ratio (SNR) of $20 \mathrm{~dB}$. The SNR is defined as

$$
S N R=10 \log \frac{P_{y_{u}}}{P_{v}}
$$

where $P_{v}$ represents the average power of the zero-mean additive noise on the system output (i.e. the variance) while $P_{y_{u}}$ denotes the average power of the noise-free output fluctuations. The noisy system response, along with the two PRBS inputs, are displayed in Figure 3(a).

The estimation results ${ }^{12}$ from a Monte Carlo simulation with $N_{\text {exp }}=200$ experiments are shown in Table 1. The aim here is first to illustrate the relevance of the proposed approach dedicated

\footnotetext{
${ }^{11}$ This algorithm is also available in the CONTSID toolbox where the parameters of MISO models are estimated by using the Levenberg-Marquardt algorithm via sensitivity functions.

${ }^{12}$ All of the identification results were computed using version 4.0 of the CONTSID toolbox on Matlab 7.0.1.
} 
to MISO transfer function model with different denominators. The estimation results obtained with the proposed SRIVC and with the traditional Instrumental Variable-based State Variable Filter (IVSVF) ${ }^{13}$ method (Garnier et al. 2003) for MISO transfer function models with common denominators are presented in Table 1 . The estimation results obtained with the COE method ${ }^{14}$ are also included in Table 1 for comparison purposes. Note that both SRIVC and COE routines are initialised in the same way from an initial estimate obtained by using the IV-based GPMF algorithm (see (Garnier et al. 2003) for example) since the automatic option based on the use of the discrete-time version SRIV is not available in the COE method.

To compare the statistical performance of the different approaches, the computed mean $\overline{\hat{\theta}}_{i, l}$ and standard deviation $\sigma_{\hat{\theta}_{i, l}}$ of the estimated parameters are presented, as well as the empirical normalised mean square error (NMSE) which is defined as

$$
\operatorname{NMSE}\left(\hat{\theta}_{i, l}\right)=\frac{1}{N_{e x p}} \sum_{j=1}^{N_{\exp }}\left(\frac{\theta_{i, l}^{o}-\hat{\theta}_{i, l}(j)}{\theta_{i, l}^{o}}\right)^{2},
$$

where $\hat{\theta}_{i, l}(j)$ is the $l$ th element of the estimated parameter vector at the $j$ th Monte Carlo simulation experiment $\hat{\theta}_{i}(j)$ ( $i=1$ or 2 here) while 'o' denotes the true value of the parameter. The average iteration number $\bar{N}_{i t e r}$, the standard deviation of iterations $\sigma_{N_{i t e r}}$ and the average computational time $\bar{T}_{c}$ for the iterative methods to converge are also considered later in the analysis of the estimation results.

The comparison of the estimation results displayed in Table 1 obtained by the SRIVC and IVSVF methods show, as expected, the relevance of the proposed SRIVC algorithm dedicated to different denominator transfer function models. It can be seen that the IVSVF method assuming common denominators fails to give a good estimate because the same dynamic is used for the two transfer functions. In contrast to this, the SRIVC algorithm, which considers different denominators, gives very accurate results with no bias and very low standard errors. This analysis is further illustrated by the Bode plots of the 200 estimated models for both SRIVC and IVSVF estimation techniques displayed in Figure 4.

Table 1 shows also that, for this system with quite similar dynamic characteristics, there is nothing to choose between the SRIVC and COE methods. The two methods dedicated to different denominator transfer function model identification are consistent with very low estimated standard errors and give their best performance when applied to simulated data that conform with the assumptions made in their derivation, which is clearly the case in this additive Gaussian measurement noise example.

\subsection{Simulation example 2}

The second system considered (S2) is also a two input, one output system given by

$$
\mathrm{S} 2:\left\{\begin{aligned}
y_{u}(t) & =\frac{-0.5 p+1}{p^{2}+0.6 p+1} u_{1}(t)+\frac{100}{p^{2}+8 p+100} u_{2}(t) \\
y\left(t_{k}\right) & =y_{u}\left(t_{k}\right)+v\left(t_{k}\right)
\end{aligned}\right.
$$

The first transfer function is identical to the one used in S1, while the second transfer function is now a minimum phase, second order which has a resonant mode, with a damping coefficient of 0.4

\footnotetext{
${ }^{13}$ This algorithm is also available in the CONTSID toolbox.

${ }^{14}$ Both SRIVC and COE iterative searches are automatically stopped by using the procedure presented in Section 3.3. (see $(33))$.
} 
and a natural frequency of $10 \mathrm{rad} / \mathrm{s}$. The dynamic characteristics between the two inputs and the output are now clearly different, as it may be seen from the step responses displayed in Figure 2(b).

The simulation conditions are the same than before. The input signal $u_{2}$ was kept at 1 for some decades of seconds after the PRBS sequence had finished ${ }^{15}$. The noisy system response, along with the two PRBS inputs, are displayed in Figure 3(b). The estimation results obtained with the proposed SRIVC and COE algorithms from a Monte Carlo simulation with 200 realizations are shown in Table 2. From this table, the difference in performance between the two estimation approaches is clearly noticeable. While the proposed SRIVC method still delivers very good estimation results, the output error-based technique is not able to converge to the global minimum for this system with quite different dynamic characteristics ${ }^{16}$. The COE iterations stop when the maximum number of iterations is achieved. Note that both algorithms are initialized in the same way from an initial IVGPMF-based model estimates. The difference in performance of both methods cannot, therefore, come from the algorithm initialization stage. The Bode plots of the 200 estimated models for both estimation techniques are plotted in Figure 5 and further illustrate the previous analysis.

\subsection{Model order selection}

The model order selection procedure presented in Section 3.4 is now applied to the second simulated system $\mathrm{S} 2$ for the same simulation conditions (same PRBS, SNR=20dB). The procedure implemented in the CONTSID SRIVCSTRUC routine is applied to search all models in the range $\left[m_{1}, m_{2}, n_{1}, n_{2}\right]=[0,0,1,1]$ to $[1,1,3,3]$. Table 3 shows the best 15 model orders sorted in increasing $Y I C$.

For this simulation example, the proposed model order estimation procedure, based on the selection of the most negative $Y I C$ and a relatively high value of $R_{T}^{2}$, clearly identifies the true model structure $\left[m_{1}, m_{2}, n_{1}, n_{2}\right]=[1,0,2,2]$. It presents the most negative $Y I C=-13.71$ with a $R_{T}^{2}=0.990$ very close to the highest $R_{T}^{2}$ value ${ }^{17}$ and with the lowest number of iterations to converge.

\section{$5 \quad$ Winding process application}

\subsection{Process description}

A diagram of the winding process is presented in Figure 6. The main part of this MIMO pilot plant is a winding process composed of a plastic web and three reels. Each reel is coupled with a direct-current motor via gear reduction. The angular speed of each reel $\left(S_{1}, S_{2}, S_{3}\right)$ is measured by a tachometer, while the tensions between the reels $\left(T_{1}, T_{3}\right)$ are measured by tension meters. At a second level, each motor is driven by a local controller. Two PI control loops adjust the motor currents $\left(I_{1}\right)$ and $\left(I_{3}\right)$ and a double PI control loop drives the angular speed $\left(S_{2}\right)$. The set-points of the local controllers $\left(I_{1}^{*}, S_{2}^{*}, I_{3}^{*}\right)$ constitute the manipulated inputs of the winding system $u(t)=\left[\begin{array}{llll}I_{1}^{*}(t) & S_{2}^{*}(t) & I_{3}^{*}(t)\end{array}\right]^{T}$. Driving a winding process essentially comes down to controlling the web linear velocity and the web tensions $\left(T_{1}\right)$ and $\left(T_{3}\right)$ around a given operating point.

\footnotetext{
${ }^{15}$ This non-zero mean portion of signal was added to the PRBS in order to ensure that the COE method estimated the correct steady state gain in the Monte Carlo simulation.

${ }^{16}$ This kind of output error minimization techniques is known to encountered difficulties (linked to local minima problems) under conditions that are non-standard, such as rapidly sampled data and dominant system modes with widely different natural frequencies (see (Ljung 2003) for example). Various remedies exist for the local minima problems as, for example, the special choice of an excitation signal or the use of robust initialisation procedure. However, these special remedies have not been further investigated here.

${ }^{17}$ where $R_{T}^{2}=0.991$, but with an associated $Y I C=-6.42$. This is why it does not appear in Table 3 .
} 
Consequently, the output variables of the winding system are $y(t)=\left[\begin{array}{lll}T_{1}(t) & T_{3}(t) & S_{2}(t)\end{array}\right]^{T}$. The process is described in more detail in (Bastogne et al. 1998).

\subsection{Experiment design}

The estimation and validation data sets are displayed in Figures 7 and 8. Discrete interval binary sequences were used as input excitation signals. The sampling rate is equal to $T_{s}=10 \mathrm{~ms}$. The experimental period, around $30 \mathrm{~s}$ for the estimation data set, is short enough to overlook the changes of the winding radii $r_{1}(t)$ and $r_{3}(t)$. The mean and linear trends of the input/output signals were removed. The raw input/output data set can be found in the Matlab file winding.mat available in the CONTSID toolbox.

\subsection{Model order selection}

The SRIVC-based procedure presented in Section 3.4 has been used to determine the transfer function orders of the winding process model. For each output, a large number of models have been estimated for a wide range of model orders. The best model structures according to identification criteria $Y I C$ and $R_{T}^{2}$ are given in Table 4. Each model presented in this Table respects two conditions

- $Y I C<\min (Y I C)+3$,

- $R_{T}^{2}<\max \left(R_{T}^{2}\right)-0.01$.

It can be seen that there is still some ambiguity about which is the best model for the three outputs. In the SISO situation, the choice of the best model is usually clear cut, since the correct model's YIC is singularly most negative in relation to higher order models. However, in this MISO and real-life data situation, for a given output, some of the models within one order of the best model possess very similar $Y I C$ and $R_{T}^{2}$ criteria. In such cases, the intuitive procedure is to choose within the potential model set, the most parsimonious model with the lowest number of parameters. Models which respect the above condition have finally been selected and these are referenced by * in Table 4.

\subsection{Model identification}

The proposed multiple inputs single output estimation scheme has been implemented for each of the three outputs. The final identified transfer function matrix is:

$$
\left(\begin{array}{l}
T_{1}(s) \\
T_{3}(s) \\
S_{2}(s)
\end{array}\right)=G(s)\left(\begin{array}{c}
I_{1}^{*}(s) \\
S_{2}^{*}(s) \\
I_{3}^{*}(s)
\end{array}\right)
$$

with

$$
G(s)=\left(\begin{array}{ccc}
\frac{-35.9( \pm 1.2)}{s^{2}+9.3( \pm 0.3) s+11.5( \pm 0.4)} & \frac{-1.3( \pm 0.02)}{s+2.3( \pm 0.04)} & \frac{2.0( \pm 0.2)}{s+6.9( \pm 0.9)} \\
\frac{-4.1( \pm 0.2) s-2.7( \pm 0.2)}{s^{2}+5.6( \pm 0.3) s+12.3( \pm 0.6)} & \frac{-1.6( \pm 0.08)}{s+6.0( \pm 0.3)} & \frac{4.8( \pm 0.1)}{s+3.7( \pm 0.1)} \\
\frac{1.1( \pm 0.05)}{s^{2}+1.4( \pm 0.1) s+7.8( \pm 0.2)} & \frac{3.4( \pm 0.02)}{s+2.7( \pm 0.01)} & \frac{1.4( \pm 0.1)}{s+5.8( \pm 0.4)}
\end{array}\right)
$$

where s denotes the Laplace variable and the figures in parentheses are the estimated standard errors. 


\subsection{Cross-validation results}

To evaluate the quality of the estimated transfer function models, a cross-validation procedure has been applied to data that were not used to build the model. Cross-validation results are plotted in Figure 9, where it may be observed that there is a satisfactory reproduction of the multiple output behaviour by the transfer function models. These results demonstrate the applicability of the proposed SRIVC algorithm for the identification of reduced-order, continuous-time, multiple transfer function models. Note that the oscillatory character of the output $\left(T_{3}\right)$ is not modeled as well as the first two outputs. However, this problem is not due to the estimation algorithm. Indeed, it has been shown in (Bastogne and Sibille 1998) that the tension $\left(T_{3}\right)$ has non linear transient behaviour depending on the sign of the steps on the input $\left(I_{1}^{*}\right)$. These input dependent dynamics cannot, therefore, be captured by a linear model identification procedure, although they may be captured if a nonlinear input transformation and this is being investigated using the state dependent parameter estimation approach to modelling nonlinear systems (see e.g. Young et al, 2001).

\section{Industrial distillation column application}

\subsection{Column description}

Figure 10 shows a schematic description of the industrial binary distillation column. It is equipped with 48 trays, a steam-heated reboiler and a total condenser. The column is fed in at the $18^{\text {th }}$ tray with a binary mixture of carbonate components. The separation of components takes place under controlled pressure. The objectives are to control the impurity of the top product or distillate $X_{t}$ and the impurity of the bottom product or residue $X_{b}$ with respect to changes on reflux flow $F_{r}$ and heating power $Q$ while preventing influence of changes on feed flow $F_{f}$ and feed composition. The distillate and residue $X_{t}$ and $X_{b}$ are measured by means of analyzers and expressed in volume per million (vpm). The process is described in more detail in (Defranoux et al. 2000).

\subsection{Experiment design}

Two kinds of experiment were carried out while respecting constraints imposed by the industrial company. These constraints were first to not perturb the production, since the top composition is a finished product; and secondly to manipulate the inputs separately for security and productivity reasons. This latter constraint required that the inputs were perturbed separately and that MISO identification was utilized. The sampling time was set to $10 \mathrm{~s}$. The two experiments, therefore, consisted of manipulating separately the set-points of the reflux flow, $F_{r}$, and the temperature of tray $40, T_{40}$, around their normal operating point; the other variables being locally controlled. The experiment lasted between 5 and 17 hours. The manipulated variables were chosen as zero-mean Random Binary Signals (RBS). Two RBS with a magnitude of $0.3 \mathrm{t} / \mathrm{h}$ and of $1.5 \mathrm{C}$ were separately applied to the reflux flow $F_{r}$ and to the temperature $T_{40}$, respectively, as illustrated in Figure 11. Before executing the estimation procedure, classical data pre-processing was carried out on the raw data sets.

\subsection{Model structure selection}

Based on detailed data analysis, it turns out that the temperature measurement of the sensitive tray $T_{12}$ can be considered as a continuous indication of the distillate $X_{t}$ that reacts quickly to changes. This sensitive tray temperature $T_{12}$ has, therefore, been considered as an output variable 
instead of the distillate. However, no temperature tray could represent a continuous indication of the residue $X_{b}$, which constitutes the second output of the model. Classically, the reflux flow $F_{r}$ and heating power represented by the controlled temperature $T_{40}$ are used as input variables for the system. The most important disturbance entering this distillation column is a change in the feed flow rate. Since the feed flow rate $F_{f}$ is measured, therefore, it has been included as a third input variable for the model. The multivariable coupling in the process can be then be described by the following model:

$$
\left(\begin{array}{c}
T_{12}(s) \\
X_{b}(s)
\end{array}\right)=\left(\begin{array}{lll}
H_{11}(s) & H_{12}(s) & H_{13}(s) \\
H_{21}(s) & H_{22}(s) & H_{23}(s)
\end{array}\right)\left(\begin{array}{c}
F_{r}(s) \\
T_{40}(s) \\
F_{f}(s)
\end{array}\right)
$$

\subsection{Model identification}

The measured reflux flow and tray 40 temperature rather than the set-points for these variables were considered in the identification procedure. Time delays from input to output variables and model orders were previously estimated from step responses and from previous identification (Defranoux et al. 2000) respectively. During the experiment, the feed flow changes did not disturb explicitly the bottom product composition of the column. The distant position of the feed tray (Figure 10) in respect to the bottom of the column probably explains this phenomenon. The transfer function $H_{23}$ was not, therefore, considered in the estimation procedure and was set to zero. Furthermore, no coupling between the reflux flow $F_{r}$ and the residue $X_{b}$ could be demonstrated. Consequently, the transfer function $H_{21}$ was also set to zero and was not considered in the estimation procedure. This explains why there is no cross-validation plot for $X_{b}$ in the case of excitation on the reflux flow set-point. The proposed multiple input, single output estimation scheme has been implemented for each of the two outputs.

\subsection{Cross-validation results}

Cross-validation results are presented in Figures 12 and 13. They are of identical quality to those obtained by using an indirect approach consisting first of estimating a discrete-time model by a prediction error method and then converting it into a continuous-time one (Defranoux et al. 2000). This application demonstrates further the practical applicability of the proposed scheme.

\section{Conclusions}

In this paper, an optimal IV-type method has been proposed to directly estimate asymptotically efficient estimates in multiple input, single output continuous-time models from sampled data, where the additive noise is white. This method also yields consistent and relatively low variance parameter estimates in the case of coloured noise; and it could be made statistically efficient in this situation by incorporating a discrete-time model for the coloured noise process, as in (Young et al. 2006). The proposed procedure differs from many other MISO estimation schemes in that it is applied to a MISO model with different transfer functions between the various inputs and the output. In this sense, the proposed refined IV procedure has considerable potential for practical application, particularly in those cases where the dynamic characteristics between the various inputs and the single output are quite different. Moreover, the proposed IV procedure, in contrast to alternative output-error minimization approaches, exploits the advantage of using an iterative, linear regression approach and, therefore, does not appear to suffer from the local minima problems that characterize the output-error methods in similar circumstances. Other advantages are that 
the proposed approach can easily handle non-uniformly sampled data and the refined IV method allows for the use of the YIC model structure identification criterion, which based on the properties of the instrumental product matrix and helps to identify the most appropriate model orders, prior to parameter estimation. All of these interesting properties have been illustrated via Monte Carlo simulations and the application to both a winding process and an industrial binary distillation column. Another successful application of the proposed estimation scheme to identify a two inputtwo output flexible robotic arm designed for heart-beating tracking is also reported in Cuvillon et al. (2006), demonstrating the wide practical applicability of the proposed identification approach.

\section{Acknowledgment}

The first two authors wish to thank their colleague Thierry Bastogne for supplying the winding process data.

\section{References}

Bastogne, T. and P. Sibille (1998). Identification of the input dependent dynamics of winding tensions. In: IFAC conference on system structure and control. Vol. 2. Nantes (France). pp. 255260.

Bastogne, T., H. Noura, P. Sibille and A. Richard (1998). Multivariable identification of a winding process by subspace methods for a tension control. Control Engineering practice 6(9), 10771088.

Cuvillon, L., E. Laroche, H. Garnier, J. Gangloff and M. de Mathelin (2006). Continuous-time model identification of robot flexibilities for fast visual servoing. In: Proceedings of the 14th IFAC Symposium on System Identification (SYSID'2006). Newcastle (Australia).

Defranoux, C., H. Garnier and P. Sibille (2000). Identification and control of an industrial binary distillation column: a case study. Chemical Engineering and Technology 23(9), 745-750.

Garnier, H. and L. Wang (2007). Continuous-time model identification from sampled data. Springer Verlag. To be published.

Garnier, H. and P.C. Young (2004). Time-domain approaches for continuous-time model identification from sampled data. In: Invited tutorial paper for the American Control Conference (ACC'2004). Boston, MA (USA).

Garnier, H., M. Gilson and O. Cervellin (2006). Latest developments for the Matlab CONTSID toolbox. In: Proceedings of the 14th IFAC Symposium on System Identification (SYSID'2006). Newcastle (Australia).

Garnier, H., M. Mensler and A. Richard (2003). Continuous-time model identification from sampled data. Implementation issues and performance evaluation. International Journal of Control 76(13), 1337-1357.

Gilson, M. and P. Van den Hof (2005). Instrumental variable methods for closed-loop system identification. Automatica 41(2), 241-249. 
Huselstein, E. and H. Garnier (2002). An approach to continuous-time model identification from non-uniformly sampled data. In: 41st IEEE Conference on Decision and Control (CDC'02). Las-Vegas, Nevada (USA).

Jakeman, A.J., L.P. Steele and P.C. Young (1980). Instrumental variable algorithms for multiple input systems described by multiple transfer functions. IEEE Transactions on Systems, Man, and Cybernetics SMC-10, 593-602.

Li, W., H. Raghavan and S. Shah (2003). Subspace identification of continuous time models for process fault detection and isolation. Journal of Process Control 13(5), 407-421.

Ljung, L. (1999). System identification. Theory for the user. 2nd ed.. Prentice Hall. Upper Saddle River.

Ljung, L. (2003). Initialisation aspects for subspace and output-error identification methods. In: European Control Conference (ECC'2003). Cambridge (U.K.).

Mahata, K. and H. Garnier (2006). Identification of continuous-time errors-in-variables models. Automatica.

Mensler, M., S. Joe and T. Kawabe (2006). Identification of a toroidal continuously variable transmission using continuous-time system identification methods. Control Engineering practice 14(1), 45-58.

Moussaoui, S., D. Brie and A. Richard (2005). Regularization aspects in continuous-time model identification. Automatica 41(2), 197-208.

Raghavan, H., A.K. Tangirala, R.B. Gopaluni and S.L. Shah (2006). Identification of chemical processes with irregular output sampling. Control Engineering practice 14(5), 467-480.

Rao, G.P. and H. Unbehauen (2006). Identification of continuous-time systems. IEE Proceedings Control theory and applications 153(2), 185-220.

Söderström, T. and P. Stoica (1983). Instrumental variable methods for system identification. Springer Verlag. New York.

Walter, E. and L. Pronzato (1997). Identification of parametric models from experimental data. Springer Verlag.

Wang, L., P. Gawthrop, C. Chessari, T. Podsiadly and A. Giles (2004). Indirect approach to continuous time system identification of food extruder. Journal of Process Control 14(6), 603615.

Young, P.C. (1970). An instrumental variable method for real-time identification of a noisy process. Automatica 6, 271-287.

Young, P.C. (1976). Some observations on instrumental variable methods of time-series analysis. International Journal of Control 23, 593-612.

Young, P.C. (1984). Recursive estimation and time-series analysis. Springer-Verlag. Berlin.

Young, P.C. (1989). Control and Dynamic Systems: Advances in Theory and Applications. Chap. Recursive estimation, forecasting and adaptive control, pp. 119-166. C.T. Leondes ed.. Academic Press. 
Young, P.C. (1996). Identification, estimation and control of continuous-time and delta operator systems. In: Conference on Identification of Engineering Systems. Swansea (Wales). pp. 1-17.

Young, P.C. (1998). Data-based mechanistic modeling of environmental, ecological, economic and engineering systems. Journal of Modelling $\& 5$ Software 13, 105-122.

Young, P.C. (2002). Optimal IV identification and estimation of continuous-time TF models. In: 15th Triennial IFAC World Congress on Automatic Control. Barcelona (Spain).

Young, P.C., A.J. Jakeman and R. McMurtries (1980). An instrumental variable method for model order identification. Automatica 16, 281-296.

Young, P.C. and A.J. Jakeman (1979). Refined instrumental variable methods of time-series analysis: Part I, SISO systems. International Journal of Control 29, 1-30.

Young, P.C. and A.J. Jakeman (1980). Refined instrumental variable methods of time-series analysis: Part III, extensions. International Journal of Control 31, 741-764.

Young, P.C. and H. Garnier (2006). Identification and estimation of continuous-time data-based mechanistic (DBM) models for environmental systems. Environmental Modelling and Software 21(8), 1055-1072.

Young, P.C., H. Garnier and M. Gilson (2006). An optimal instrumental variable approach for identifying hybrid continuous-time Box-Jenkins model. In: Proceedings of the 14th IFAC Symposium on System Identification (SYSID'2006). Newcastle (Australia).

Young, P.C., P. McKenna and J. Bruun (2001). Identification of nonlinear stochastic systems by state dependent parameter estimation. International Journal of Control 74, 1837-1857. 

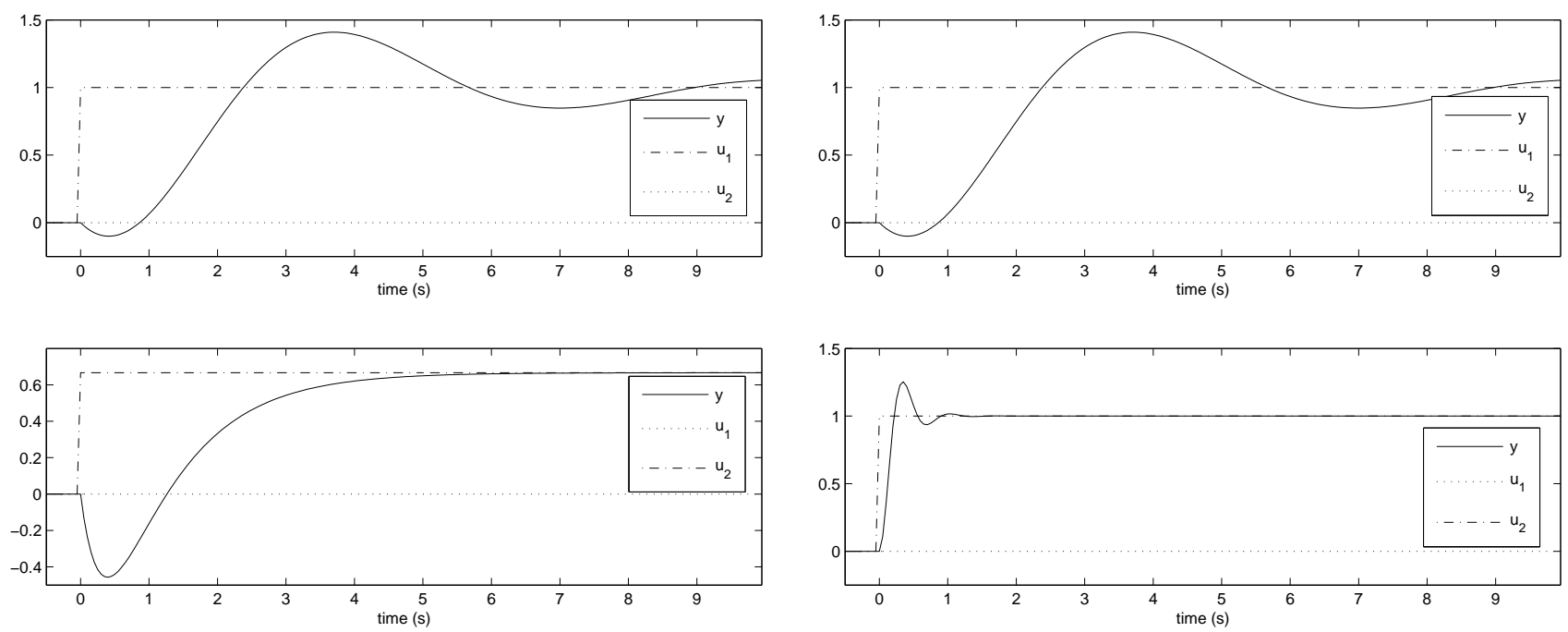

(a) System 1

(b) System 2

Figure 2: Step responses of the two inputs one output simulation systems
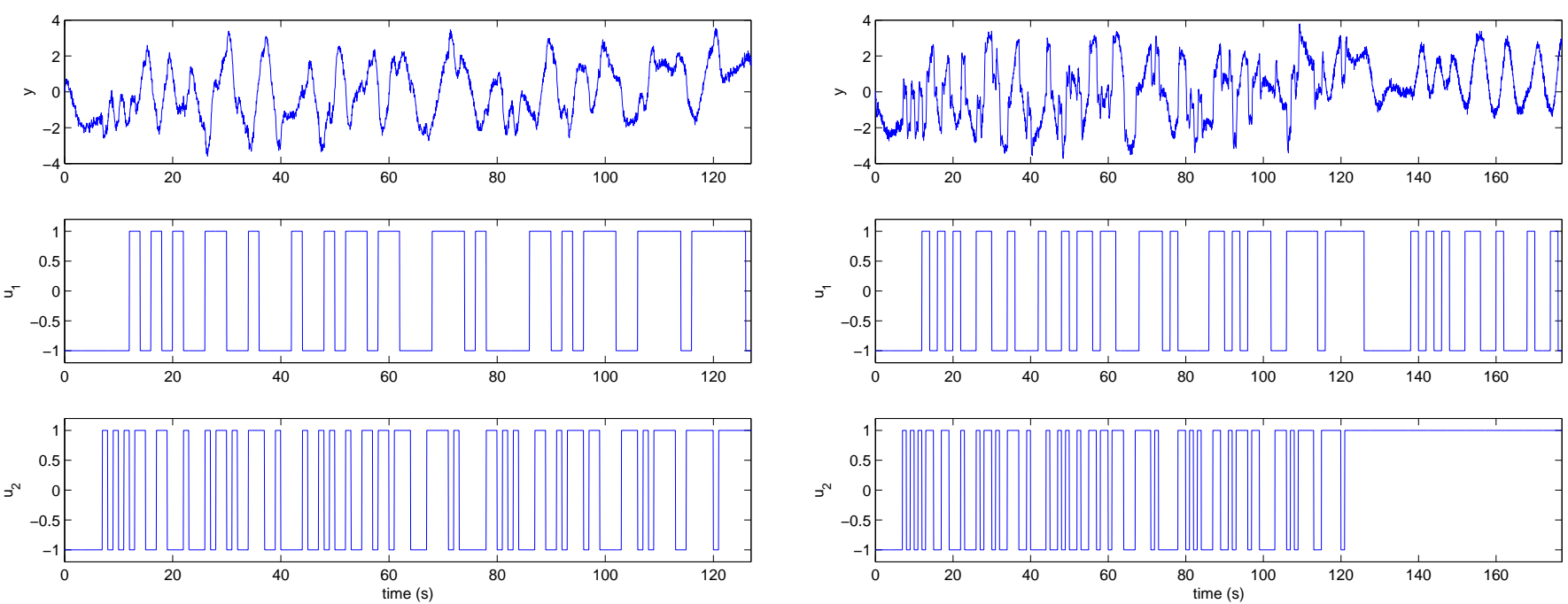

(a) System 1

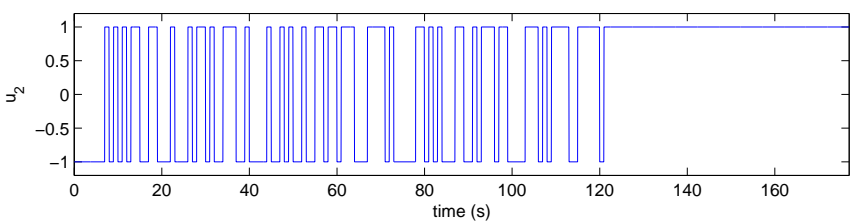

(b) System 2

Figure 3: System response to the PRBS inputs for a $S N R=20 \mathrm{~dB}$

\begin{tabular}{|c|c|c|c|c|c|c|c|c|c|c|c|}
\hline & & $b_{1,1}$ & $b_{1,0}$ & $f_{1,1}$ & $f_{1,0}$ & $b_{2,1}$ & $b_{2,0}$ & $f_{2,1}$ & $f_{2,0}$ & $\bar{N}_{i t e r} \pm \sigma_{N_{i t e r}}$ & $\bar{T}_{c}$ \\
\hline method & True value & -0.5 & 1 & 0.6 & 1 & -3 & 2 & 4 & 3 & & \\
\hline \multirow{3}{*}{ IVSVF } & $\overline{\hat{\theta}}_{i, l}$ & -1.339 & 1.747 & 1.970 & 1.737 & -1.697 & 1.873 & 1.970 & 1.737 & & \\
\hline & $\sigma_{\hat{\theta}_{i, l}}$ & 0.022 & 0.019 & 0.024 & 0.014 & 0.027 & 0.016 & 0.024 & 0.014 & & 0.44 \\
\hline & $\operatorname{NMSE}\left(\hat{\theta}_{i, l}\right)$ & 2.8 & $5.6 \mathrm{e}-1$ & 5.2 & $5.4 \mathrm{e}-1$ & $1.9 \mathrm{e}-1$ & $4.1 \mathrm{e}-3$ & $2.6 \mathrm{e}-1$ & $1.8 \mathrm{e}-1$ & & \\
\hline \multirow{3}{*}{ SRIVC } & $\overline{\hat{\theta}}_{i, l}$ & -0.500 & 1.001 & 0.601 & 1.000 & -3.001 & 1.999 & 4.002 & 3.001 & & \\
\hline & $\sigma_{\hat{\theta}_{i, l}}$ & 0.005 & 0.004 & 0.003 & 0.003 & 0.037 & 0.022 & 0.050 & 0.030 & $8.69 \pm 0.46$ & 4.02 \\
\hline & $\operatorname{NMSE}\left(\hat{\theta}_{i, l}\right)$ & $9.5 \mathrm{e}-5$ & $1.8 \mathrm{e}-5$ & $3.3 e-5$ & $8.2 \mathrm{e}-6$ & $1.5 \mathrm{e}-4$ & $1.3 \mathrm{e}-4$ & $1.6 \mathrm{e}-4$ & $1.0 \mathrm{e}-4$ & & \\
\hline \multirow{3}{*}{$\mathrm{COE}$} & $\overline{\hat{\theta}}_{i, l}$ & -0.500 & 1.001 & 0.601 & 1.000 & -3.001 & 1.999 & 4.002 & 3.001 & & \\
\hline & $\sigma_{\hat{\theta}_{i, l}}$ & 0.005 & 0.004 & 0.003 & 0.003 & 0.037 & 0.022 & 0.050 & 0.030 & $6.46 \pm 0.66$ & 6.09 \\
\hline & $\operatorname{NMSE}\left(\hat{\theta}_{i, l}\right)$ & $9.5 \mathrm{e}-5$ & $1.8 \mathrm{e}-5$ & $3.3 e-5$ & $8.2 \mathrm{e}-6$ & $1.5 \mathrm{e}-4$ & $1.3 \mathrm{e}-4$ & $1.6 \mathrm{e}-4$ & $1.0 \mathrm{e}-4$ & & \\
\hline
\end{tabular}

Table 1: Monte Carlo simulation results for System 1 

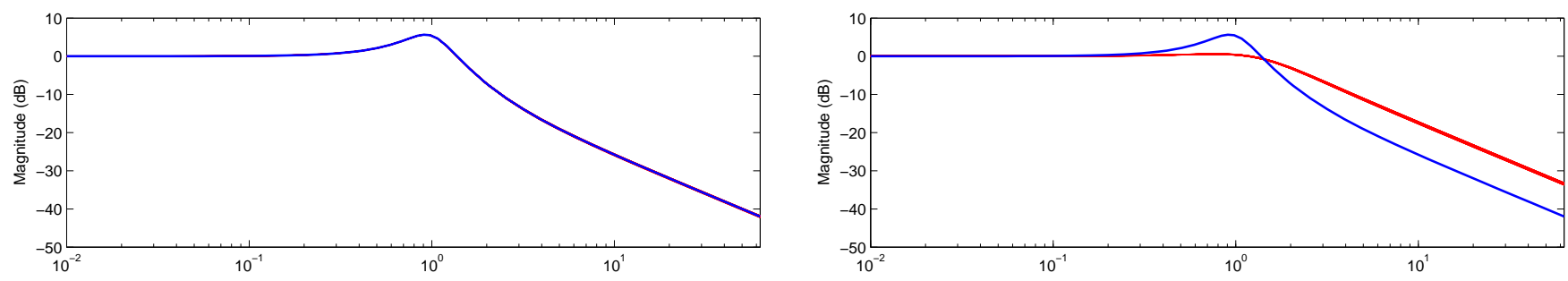

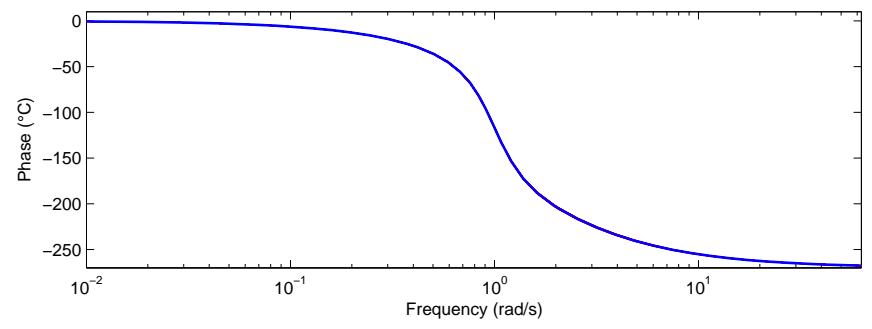

(a) Estimated $G_{1}(s)$ by SRIVC
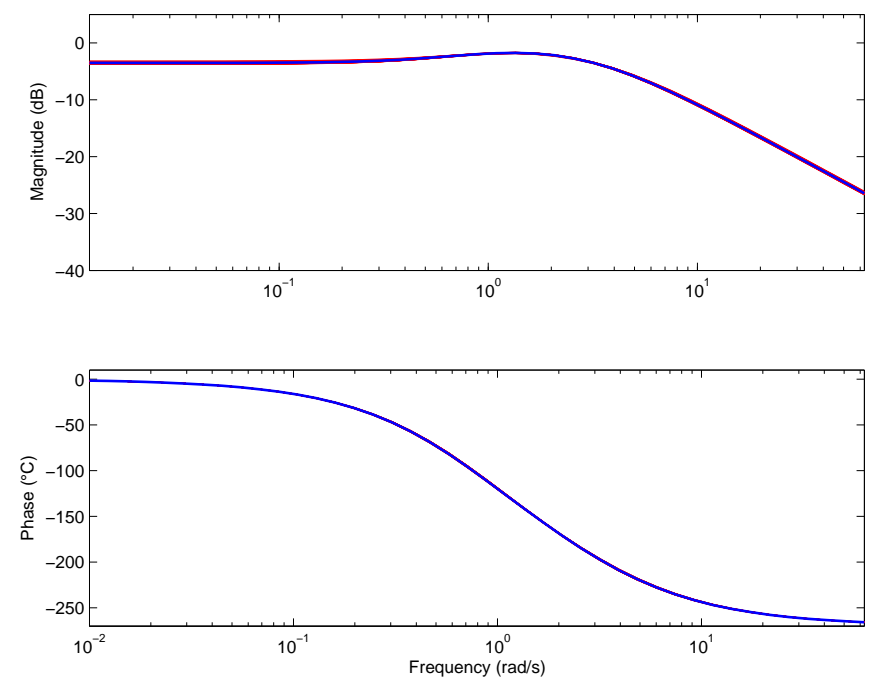

(c) Estimated $G_{2}(s)$ by SRIVC

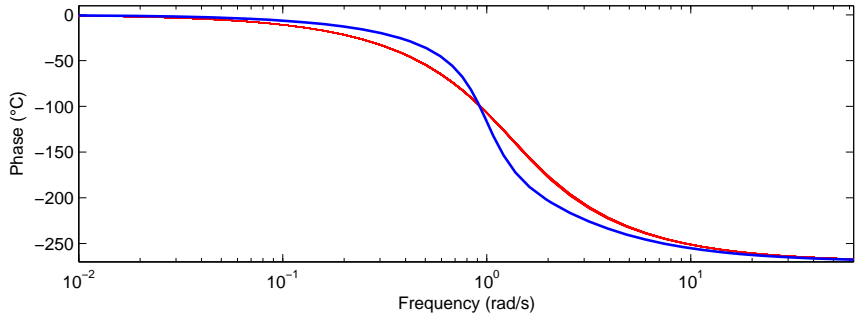

(b) Estimated $G_{1}(s)$ by IVSVF
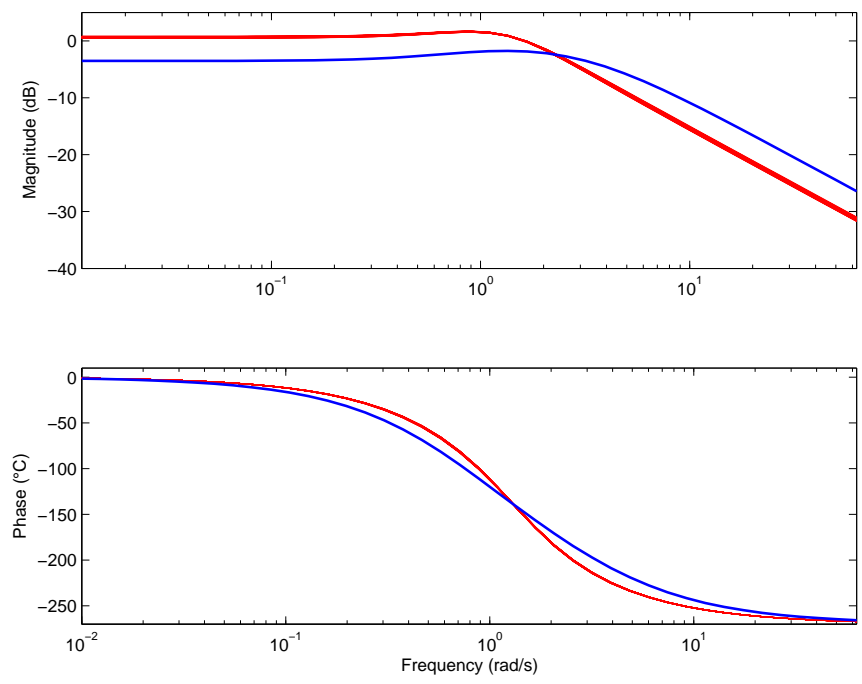

(d) Estimated $G_{2}(s)$ by IVSVF

Figure 4: Bode plots of estimated SRIVC and IVSVF models for System 1

\begin{tabular}{|c|c|c|c|c|c|c|c|c|c|c|}
\hline & & $b_{1,1}$ & $b_{1,0}$ & $f_{1,1}$ & $f_{1,0}$ & $b_{2,0}$ & $f_{2,1}$ & $f_{2,0}$ & $\bar{N}_{i t e r} \pm \sigma_{N_{i t e r}}$ & $\bar{T}_{c}$ \\
\hline method & True value & -0.5 & 1 & 0.6 & 1 & 100 & 8 & 100 & & \\
\hline \multirow{3}{*}{ SRIVC } & $\overline{\hat{\theta}}_{i, l}$ & -0.500 & 1.000 & 0.600 & 1.000 & 99.947 & 7.995 & 99.944 & & \\
\hline & $\sigma_{\hat{\theta}_{i, l}}$ & 0.005 & 0.004 & 0.003 & 0.003 & 1.134 & 0.121 & 1.031 & $6.65 \pm 0.56$ & 2.99 \\
\hline & $\operatorname{NMSE}\left(\hat{\theta}_{i, l}\right)$ & $1.2 \mathrm{e}-4$ & $1.8 \mathrm{e}-5$ & $2.8 \mathrm{e}-5$ & $7.3 \mathrm{e}-6$ & $1.3 \mathrm{e}-4$ & $2.3 \mathrm{e}-4$ & $1.1 \mathrm{e}-4$ & & \\
\hline \multirow{3}{*}{$\mathrm{COE}$} & $\overline{\hat{\theta}}_{i, l}$ & -0.400 & 0.986 & 0.583 & 0.978 & $3.4 \mathrm{e}+6$ & $2.6 \mathrm{e}+6$ & $4.4 \mathrm{e}+6$ & & \\
\hline & $\sigma_{\hat{\theta}_{i, l}}$ & 0.011 & 0.005 & 0.004 & 0.003 & $1.8 \mathrm{e}+6$ & $1.5 \mathrm{e}+6$ & $2.4 \mathrm{e}+6$ & $50 \pm 0$ & 19.77 \\
\hline & $\operatorname{NMSE}\left(\hat{\theta}_{i, l}\right)$ & $4.1 \mathrm{e}-2$ & $2.1 \mathrm{e}-4$ & $8.7 \mathrm{e}-4$ & $4.9 \mathrm{e}-4$ & $1.5 \mathrm{e}+9$ & $1.4 \mathrm{e}+11$ & $2.5 \mathrm{e}+9$ & & \\
\hline
\end{tabular}

Table 2: Monte Carlo simulation results for System 2 

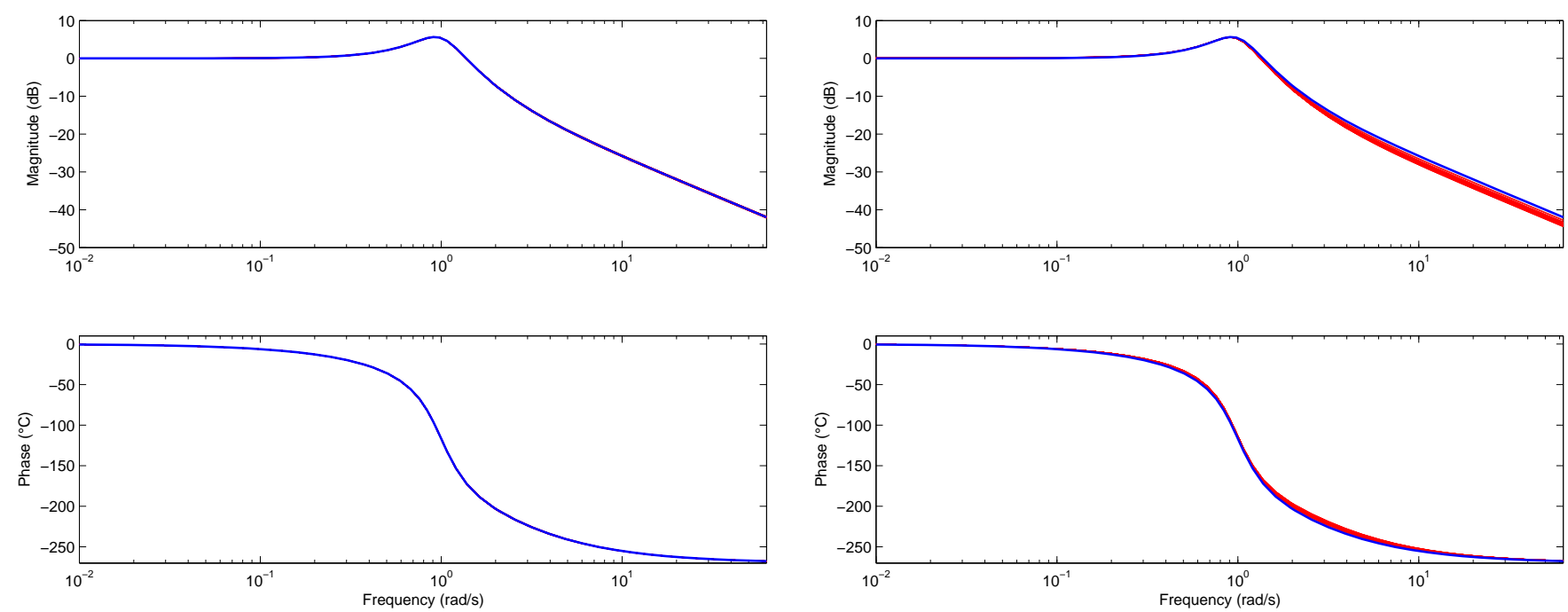

(a) Estimated $G_{1}(s)$ by SRIVC

(b) Estimated $G_{1}(s)$ by $\mathrm{COE}$
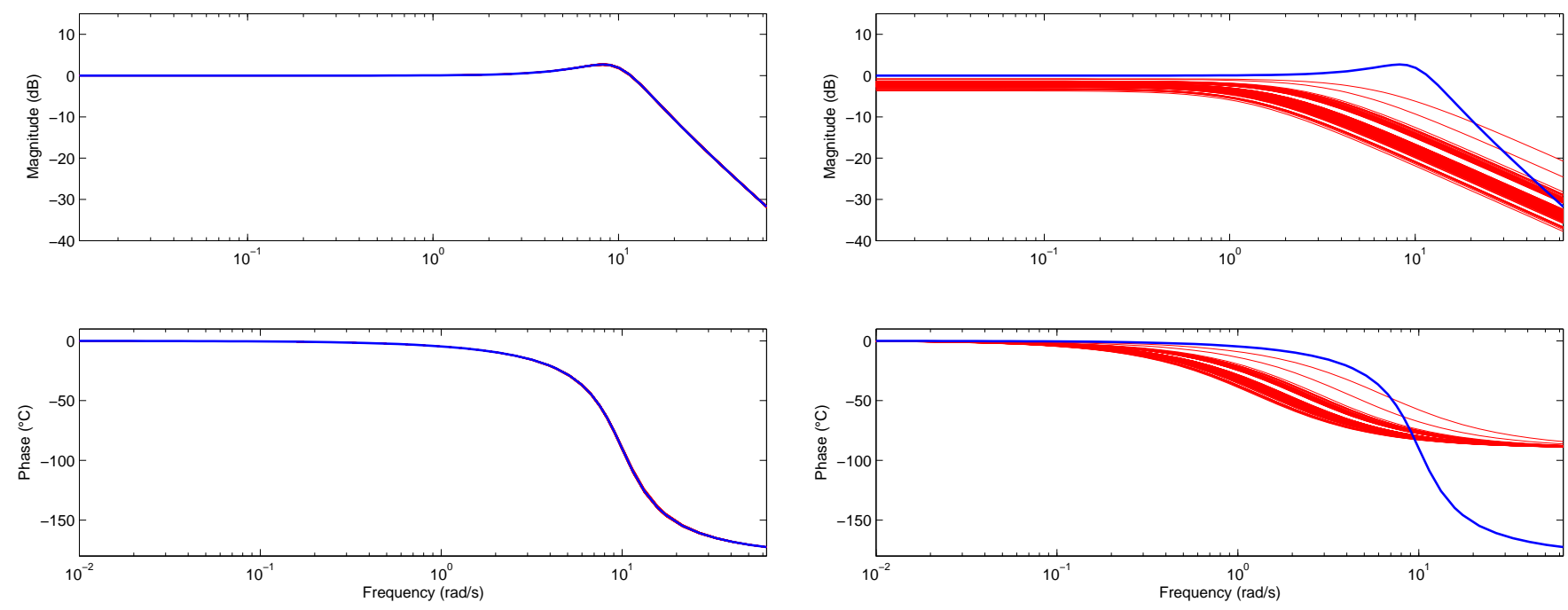

(c) Estimated $G_{2}(s)$ by SRIVC

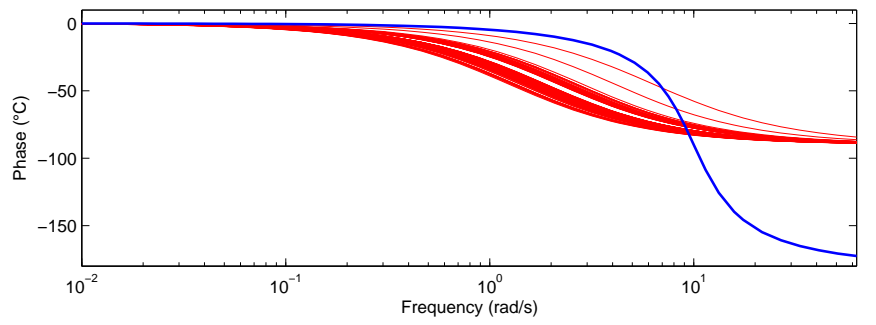

(d) Estimated $G_{2}(s)$ by $\mathrm{COE}$

Figure 5: Bode plots of estimated SRIVC and COE models for System 2 


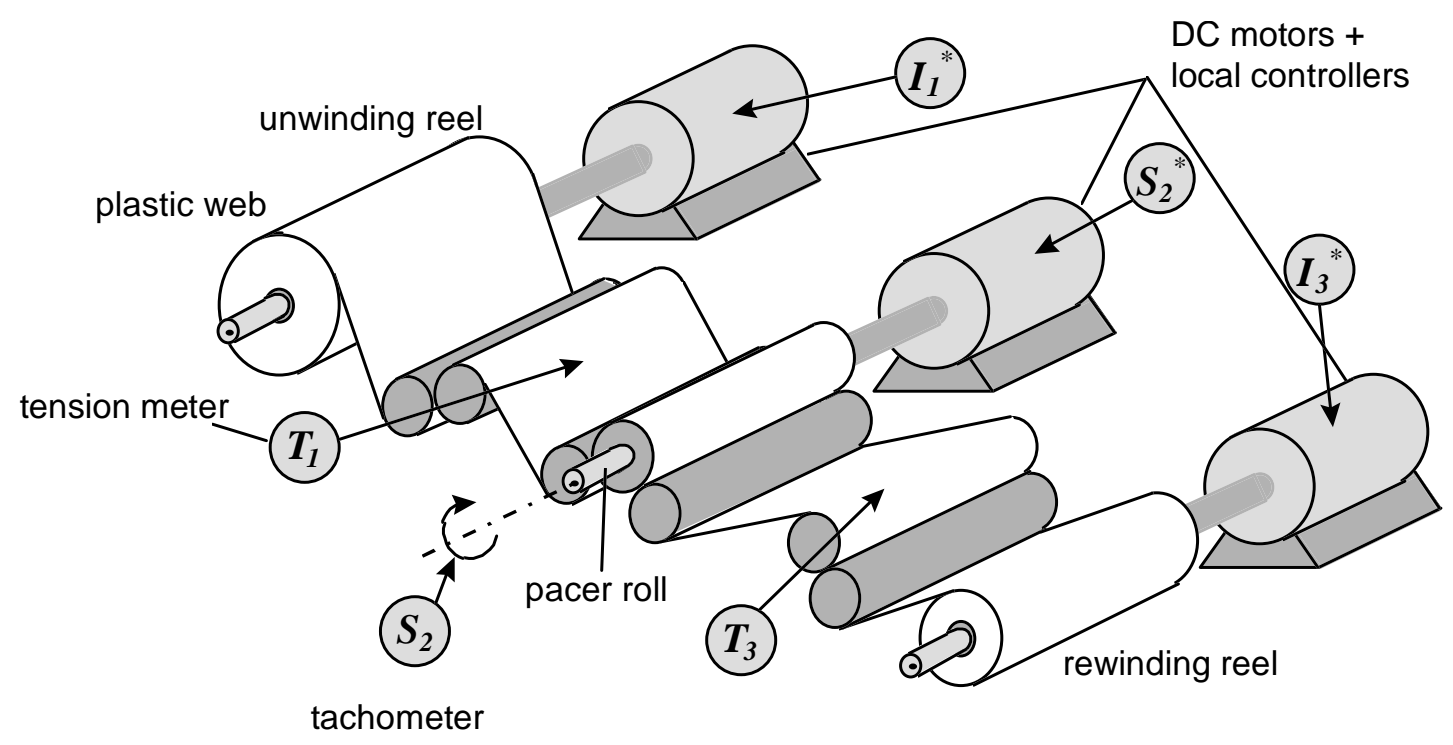

Figure 6: Winding process diagram

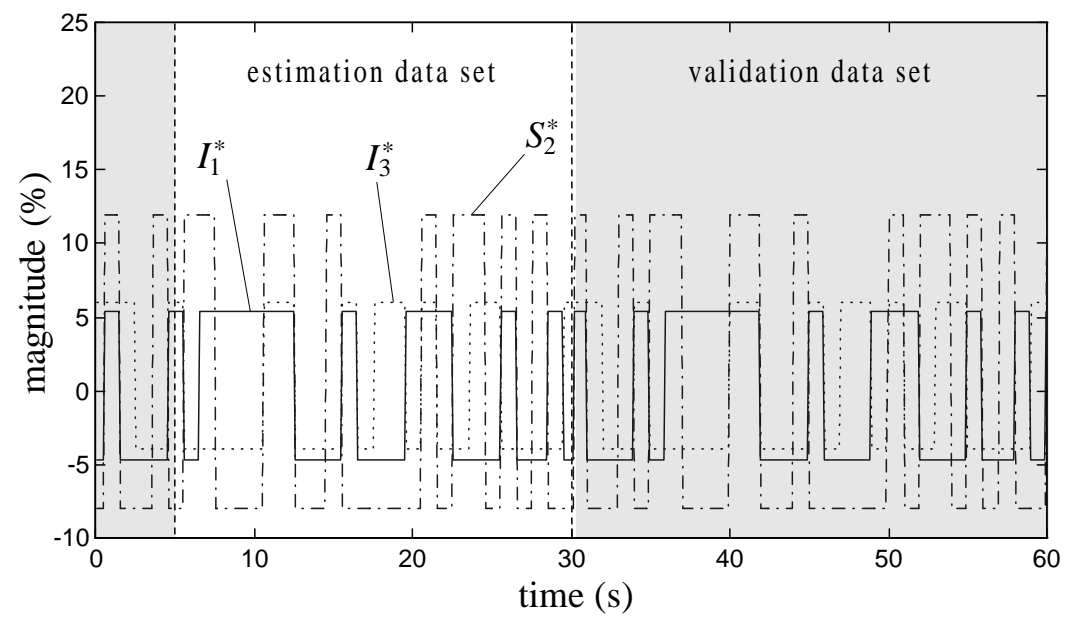

Figure 7: Input data

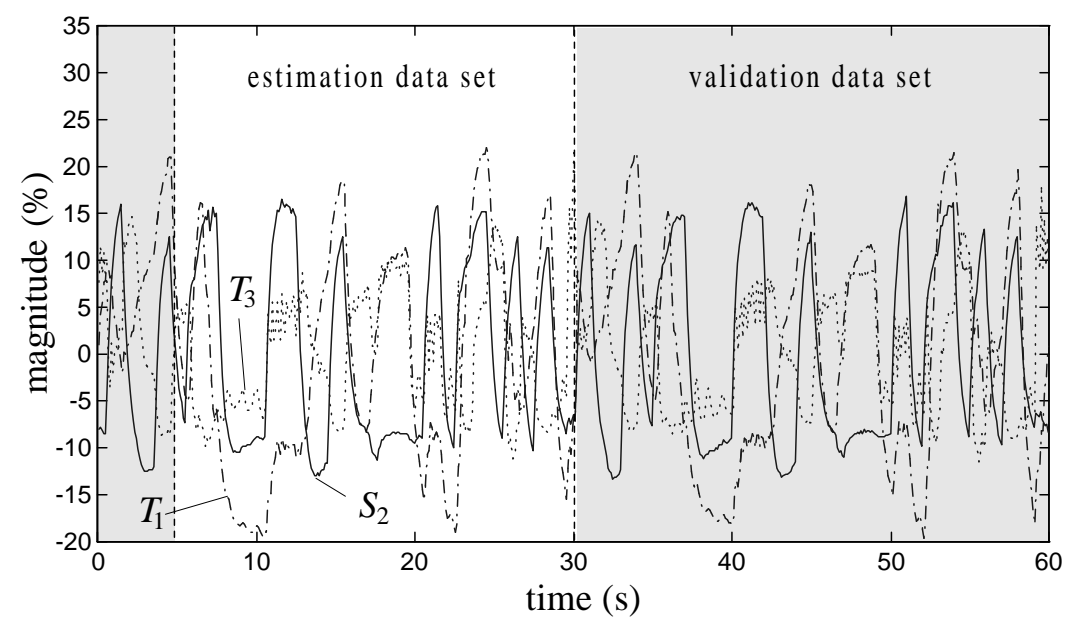

Figure 8: Output data 

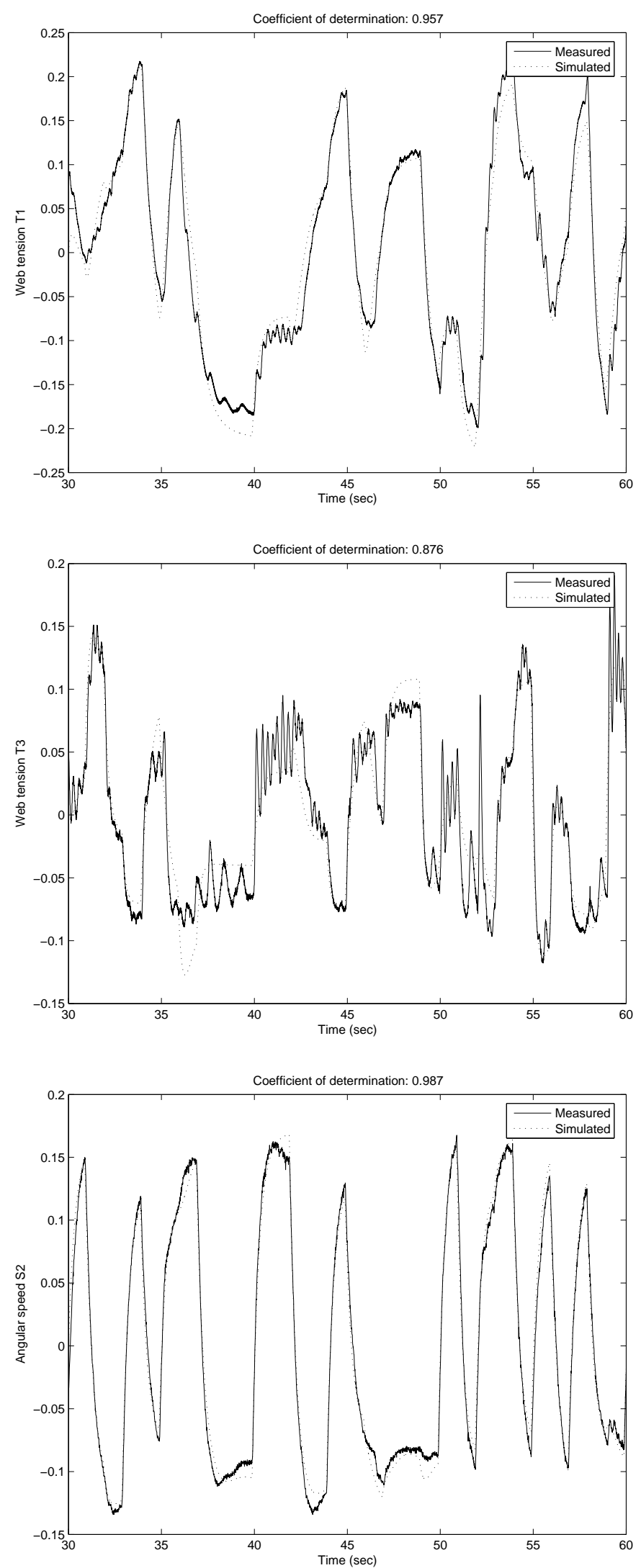

Figure 9: Cross-validation results for the winding process 


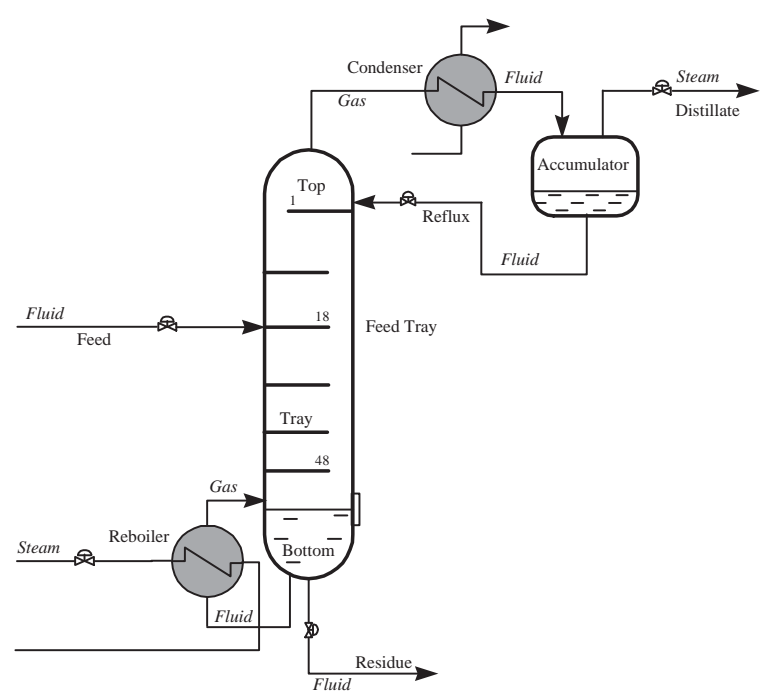

Figure 10: Schematic description of the column
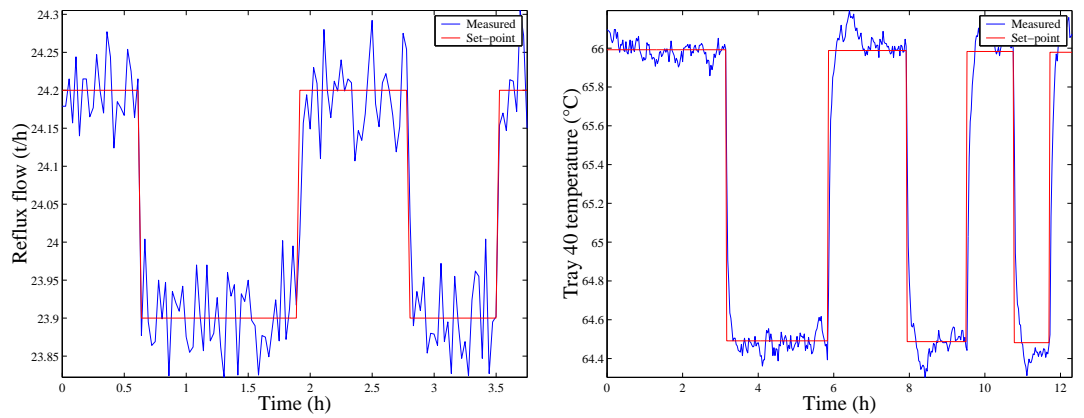

Figure 11: RBS excitation signals

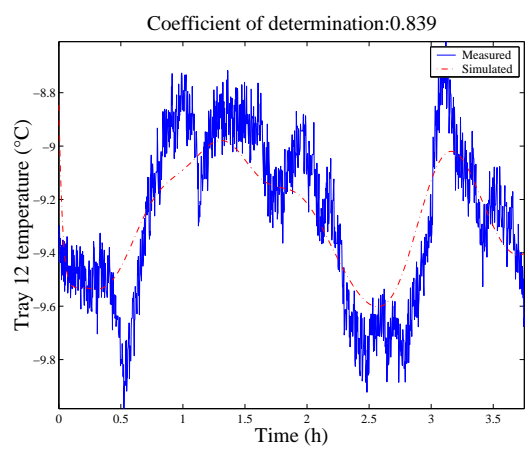

Figure 12: Cross-validation results for RBS excitation on the reflux flow set-point 

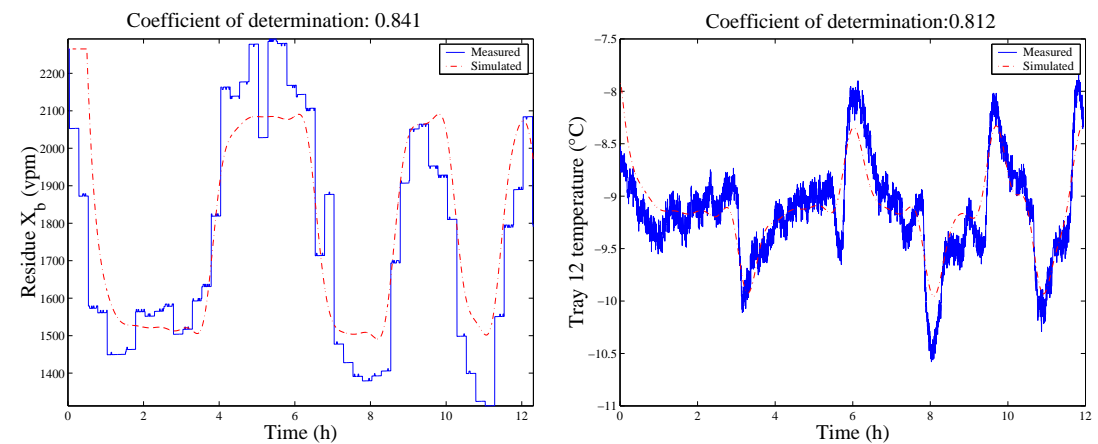

Figure 13: Cross-validation results for RBS excitation on the tray 40 temperature set-point

\begin{tabular}{ccccccc}
$m_{1}$ & $m_{2}$ & $n_{1}$ & $n_{2}$ & YIC & $R_{T}^{2}$ & $N_{\text {iter }}$ \\
\hline \hline 1 & 0 & 2 & 2 & -13.71 & 0.990 & 6 \\
1 & 0 & 2 & 1 & -12.03 & 0.975 & 17 \\
1 & 1 & 2 & 1 & -12.03 & 0.975 & 17 \\
0 & 0 & 3 & 1 & -11.17 & 0.970 & 17 \\
0 & 1 & 3 & 1 & -11.17 & 0.970 & 17 \\
0 & 0 & 2 & 2 & -11.06 & 0.959 & 9 \\
0 & 0 & 2 & 1 & -10.50 & 0.945 & 21 \\
0 & 1 & 2 & 1 & -10.50 & 0.945 & 21 \\
0 & 1 & 3 & 3 & -7.92 & 0.985 & 21 \\
0 & 0 & 1 & 2 & -7.08 & 0.738 & 50 \\
1 & 0 & 1 & 2 & -7.08 & 0.738 & 50 \\
0 & 0 & 1 & 1 & -6.96 & 0.722 & 50 \\
1 & 0 & 1 & 1 & -6.96 & 0.722 & 50 \\
0 & 1 & 1 & 1 & -6.96 & 0.722 & 50 \\
1 & 1 & 1 & 1 & -6.96 & 0.722 & 50
\end{tabular}

Table 3: Best 15 model orders according to $Y I C$ and $R_{T}^{2}$ for System 2 


\begin{tabular}{|c|c|c|c|c|c|c|c|}
\hline$m_{1}$ & $m_{2}$ & $m_{3}$ & $n_{1}$ & $n_{2}$ & $n_{3}$ & $Y I C$ & $R_{T}^{2}$ \\
\hline \multicolumn{8}{|c|}{ Output $1, T_{1}\left(t_{k}\right)$} \\
\hline 0 & 0 & 1 & 2 & 1 & 2 & -8.96 & 0.960 \\
\hline 1 & 0 & 1 & 1 & 1 & 2 & -8.82 & 0.957 \\
\hline 0 & 0 & 1 & 2 & 2 & 2 & -8.78 & 0.962 \\
\hline 0 & 0 & 0 & 2 & 1 & 1 & $-8.68^{*}$ & 0.955 \\
\hline 1 & 0 & 0 & 1 & 2 & 2 & -8.57 & 0.953 \\
\hline 1 & 0 & 1 & 1 & 2 & 2 & -8.53 & 0.958 \\
\hline 0 & 1 & 1 & 2 & 2 & 2 & -8.33 & 0.962 \\
\hline 0 & 1 & 1 & 2 & 1 & 2 & -8.29 & 0.960 \\
\hline 0 & 1 & 1 & 1 & 2 & 2 & -8.25 & 0.953 \\
\hline \multicolumn{8}{|c|}{ Output $2, T_{3}\left(t_{k}\right)$} \\
\hline 1 & 0 & 0 & 2 & 1 & 1 & $-8.03^{*}$ & 0.882 \\
\hline 1 & 0 & 0 & 2 & 2 & 1 & -7.81 & 0.884 \\
\hline 1 & 0 & 1 & 2 & 2 & 1 & -7.32 & 0.885 \\
\hline 1 & 0 & 1 & 2 & 1 & 1 & -7.28 & 0.883 \\
\hline 1 & 1 & 0 & 2 & 2 & 1 & -7.26 & 0.890 \\
\hline 1 & 1 & 1 & 2 & 2 & 1 & -6.86 & 0.891 \\
\hline 1 & 0 & 0 & 2 & 1 & 2 & -6.74 & 0.884 \\
\hline \multicolumn{8}{|c|}{ Output $3, S_{2}\left(t_{k}\right)$} \\
\hline 0 & 1 & 0 & 2 & 2 & 1 & -10.52 & 0.988 \\
\hline 0 & 1 & 0 & 2 & 1 & 1 & -10.34 & 0.985 \\
\hline 0 & 0 & 0 & 2 & 2 & 1 & -10.30 & 0.986 \\
\hline 0 & 0 & 0 & 2 & 1 & 1 & $-10.14^{*}$ & 0.983 \\
\hline 0 & 0 & 0 & 2 & 2 & 2 & -9.93 & 0.986 \\
\hline 0 & 1 & 1 & 2 & 2 & 1 & -9.93 & 0.988 \\
\hline 1 & 0 & 1 & 2 & 1 & 2 & -9.71 & 0.986 \\
\hline 1 & 1 & 1 & 2 & 1 & 2 & -9.70 & 0.987 \\
\hline 1 & 0 & 0 & 2 & 1 & 1 & -9.67 & 0.983 \\
\hline 1 & 0 & 1 & 2 & 2 & 2 & -9.65 & 0.987 \\
\hline 1 & 0 & 0 & 2 & 2 & 2 & -9.61 & 0.986 \\
\hline 1 & 1 & 0 & 2 & 1 & 2 & -9.53 & 0.986 \\
\hline 0 & 0 & 1 & 2 & 2 & 2 & -9.26 & 0.987 \\
\hline 0 & 0 & 0 & 2 & 1 & 2 & -9.23 & 0.983 \\
\hline
\end{tabular}

Table 4: Best model structures according to $Y I C$ and $R_{T}^{2}$ for the winding process 